\title{
ANALYSIS OF A CONTACT PROBLEM FOR A VISCOELASTIC BRESSE SYSTEM
}

\author{
Maria Inês M. Copetti ${ }^{1, *}$, Toufic EL Arwadi² ${ }^{2}$ Jose R. Fernández ${ }^{3}$, \\ Maria Grazia Naso ${ }^{4}$ and WaEl YousseF ${ }^{5}$
}

\begin{abstract}
In this paper, we consider a contact problem between a viscoelastic Bresse beam and a deformable obstacle. The well-known normal compliance contact condition is used to model the contact. The existence of a unique solution to the continuous problem is proved using the Faedo-Galerkin method. An exponential decay property is also obtained defining an adequate Liapunov function. Then, using the finite element method and the implicit Euler scheme, a finite element approximation is introduced. A discrete stability property and a priori error estimates are proved. Finally, some numerical experiments are performed to demonstrate the decay of the discrete energy and the numerical convergence.
\end{abstract}

Mathematics Subject Classification. 65M15, 65M60, 74B05, 74K10.

Received April 2, 2020. Accepted March 22, 2021.

\section{INTRODUCTION}

The last decades have witnessed a rapid development in high technologies using beams and a growing attention has been paid to the mathematical theory of contact mechanics (see, e.g., $[4,20,26]$ ). This has prompted great interest and several results have been published. In the wide literature on this field, most of papers deal with Euler-Bernoulli models, some of them analyze Timoshenko systems, and only few of them are devoted to Bresse ones.

It was proved in $[7,32]$ that the beam (plate) model of Timoshenko type has a wider range of applicability than Euler-Bernoulli model. In particular, the Timoshenko beam theory is widely used to describe the dynamics of a beam when the transverse shear strain is significant. Furthermore, if the longitudinal displacement is considered, the model becomes the Bresse system [14]. Conversely, the Euler-Bernoulli theory does not take into account

Keywords and phrases. Contact problem, Bresse beam, exponential decay, finite element discretization.

1 LANA, Departamento de Matemática, Universidade Federal de Santa Maria, 97105-900 Santa Maria, RS, Brazil.

2 Department of Mathematics and Computer Science, Faculty of Science, Beirut Arab University, Debbieh, Lebanon.

3 Departamento de Matemática Aplicada I, Universidade de Vigo, Escola de Enxeñería de Telecomunicación,

Campus As Lagoas Marcosende s/n, 36310 Vigo, Spain.

4 Dipartimento di Ingegneria Civile, Architettura, Territorio, Ambiente e di Matematica, Università degli Studi di Brescia, Via Valotti 9, 25133 Brescia, Italy.

5 Department of Mathematics, Faculty of Sciences 1, Lebanese University, Hadath, Lebanon.

*Corresponding author: mimcopetti@ufsm.br 
such effects: the limitations of the Kirchhoff and Euler-Bernoulli theories are well known, even if rotary inertia is included, and beam models involving improved theories need to be considered.

This paper focuses on phenomena related to contacts in materials of Bresse type. When the obstacles are rigid, the contact assumption can be modeled by the classical Signorini non-penetration condition (see, e.g., $[21,29])$, which also contributes a strong non-linearity to the problem.

In particular, here we consider a circular beam with radius of curvature $R$ whose reference configuration is the arc with length $L$. Let $x \in[0, L]$ denote the length along the undeformed beam. The equations governing the motion of the beam are given by a Bresse system of this following type

$$
\left.\begin{array}{ll}
\rho_{1} \varphi_{t t}-k\left(\varphi_{x}+l \omega+\psi\right)_{x}-\zeta\left(\varphi_{x}+l \omega+\psi\right)_{x t}-k_{0} l\left(\omega_{x}-l \varphi\right)-\zeta l\left(\omega_{x}-l \varphi\right)_{t}=0 & \text { in }(0, L) \times(0, \infty), \\
\rho_{2} \psi_{t t}-b \psi_{x x}-\zeta \psi_{x x t}+k\left(\varphi_{x}+\psi+l \omega\right)+\zeta\left(\varphi_{x}+l \omega+\psi\right)_{t}=0 & \text { in }(0, L) \times(0, \infty), \\
\rho_{1} \omega_{t t}-k_{0}\left(\omega_{x}-l \varphi\right)_{x}-\zeta\left(\omega_{x}-l \varphi\right)_{x t}+k l\left(\varphi_{x}+\psi+l \omega\right)+\zeta l\left(\varphi_{x}+\psi+l \omega\right)_{t}=0 & \text { in }(0, L) \times(0, \infty),
\end{array}\right\}
$$

where $\varphi$ and $\omega$ are the transverse and longitudinal displacements, respectively, and $\psi$ is the rotation angle of the filament. Here $\rho_{1}, \rho_{2}, k, k_{0}$ and $b$ are positive constants characterizing physical properties, $\zeta>0$ is a viscosity coefficient and $l=1 / R$.

We suppose that the beam is clamped at its left end $x=0$ and free to move at the end $x=L$, only in the transverse direction, where two flexible obstacles are located at distances $g_{1}>0$ and $g_{2}>0$ with gap $g=g_{1}+g_{2}$ possibly asymmetrical as in Figure 1. Thus, the boundary conditions are

$$
\left.\begin{array}{l}
\varphi(0, t)=\psi(0, t)=\omega(0, t)=0, \\
b \psi_{x}(L, t)+\zeta \psi_{x t}(L, t)=0, \\
\omega(L, t)=0 \\
\sigma(L, t)=-\frac{1}{\varepsilon}\left(\left[\varphi(L, t)-g_{2}\right]_{+}-\left[-\varphi(L, t)-g_{1}\right]_{+}\right),
\end{array}\right\}
$$

where $\sigma(x, t)=k\left(\varphi_{x}+l \omega+\psi\right)+\zeta\left(\varphi_{x}+l w+\psi\right)_{t},[f]_{+}=\max \{f, 0\}$, and $1 / \varepsilon>0$ represents the rigidity of the obstacles.

The equations are also supplemented by initial conditions:

$$
\left.\begin{array}{ll}
\omega(\cdot, 0)=\omega_{0}, \omega_{t}(\cdot, 0)=\omega_{1}, \varphi(\cdot, 0)=\varphi_{0} & \text { on }(0, L) \\
\varphi_{t}(\cdot, 0)=\varphi_{1}, \psi(\cdot, 0)=\psi_{0}, \psi_{t}(\cdot, 0)=\psi_{1} & \text { on }(0, L)
\end{array}\right\}
$$

According to the last boundary condition, it may occur that $\varphi(L, t)>g_{2}$ or $\varphi(L, t)<-g_{1}$. When $\varepsilon \rightarrow 0$ the obstacles become rigid and $-g_{1} \leq \varphi(L, t) \leq g_{2}$ modeling a part of the Signorini contact condition. Assuming $(1.2)_{4}$ we are considering a normal compliance condition (see, e.g., [31]) as a regularization of the Signorini contact condition. Actually, we relax the non-penetration condition by supposing for instance that the stops at the right end of the system are flexible.

The energy of the system (1.1)-(1.3) is given by

$$
\begin{aligned}
\mathcal{E}(t)= & \frac{1}{2} \int_{0}^{L}\left(\rho_{1}\left|\varphi_{t}\right|^{2}+\rho_{2}\left|\psi_{t}\right|^{2}+\rho_{1}\left|\omega_{t}\right|^{2}+b\left|\psi_{x}\right|^{2}+k\left|\varphi_{x}+\psi+l \omega\right|^{2}\right. \\
& \left.+k_{0}\left|\omega_{x}-l \varphi\right|^{2}+\frac{1}{\varepsilon}\left(\left[\varphi(L, t)-g_{2}\right]_{+}^{2}+\left[-g_{1}-\varphi(L, t)\right]_{+}^{2}\right)\right) \mathrm{d} x
\end{aligned}
$$

and we note that this energy is decreasing, that is,

$$
\frac{\mathrm{d}}{\mathrm{d} t} \mathcal{E}(t)=-\int_{0}^{L}\left(\zeta\left|\left(\varphi_{x}+\psi+l \omega\right)_{t}\right|^{2}+\zeta\left|\psi_{x t}\right|^{2}+\zeta\left|\omega_{x t}-l \varphi_{t}\right|^{2}\right) \mathrm{d} x \leq 0 .
$$




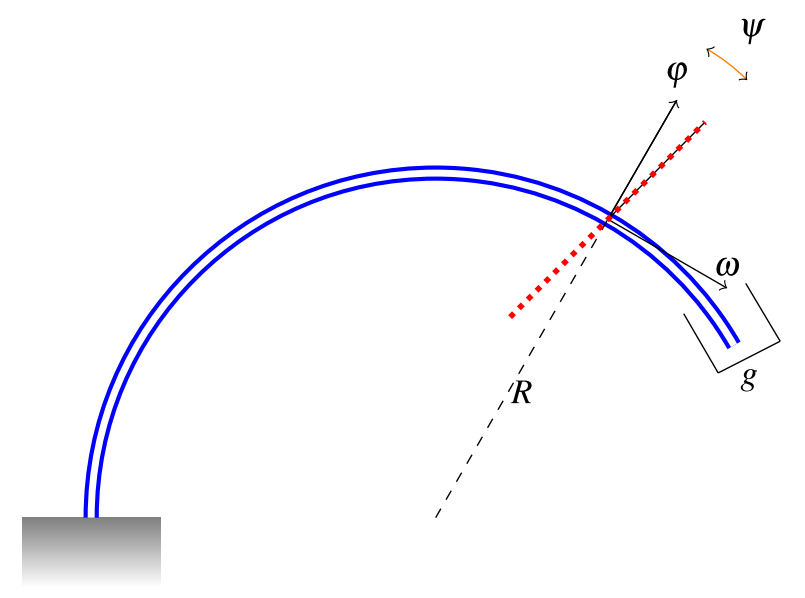

Figure 1. The circular arch and the joint with clearance $g=g_{1}+g_{2}$.

There is a large literature on the modeling, well-posedness and longtime behavior of systems in contact (see, e.g., $[22,27]$ and references therein). Applications of unilateral multibody dynamics have been analyzed in, e.g., $[36,38]$. A contact problem for a nonlinear thermoviscoelastic Timoshenko beam model was investigated theoretically and numerically by Bernardi and Copetti [8].

A first approach of research in such a context is the mathematical formulation of the contact models leading to PDE systems that are worth analyzing also regarding the existence, uniqueness, and regularity of the solutions (see, e.g., $[3,30,31]$ ), or their numerical analysis (see, e.g., $[5,6,10,15,17-19])$.

Another way of interest concerns the study of the longtime behavior of the solutions related to contact problems involving only a single displacement and/or a single variation of temperature (see, e.g., [11,35]), or referring the dynamic contact between two bodies (see, e.g., $[9,12,13,34]$ ).

The longtime behavior of Bresse systems, with different dissipative mechanism, has been considered in recent years.

The stability of the Bresse system (1.1) with Dirichlet boundary conditions was studied by El Arwadi and Youssef [23] where exponential decay was obtained without any condition on the physical constants.

In [1] the Bresse system has been investigated with frictional dissipation, present only in the equation of angular displacement. In that work, the equalities

$$
\frac{\rho_{1}}{\rho_{2}}=\frac{k}{b} \quad \text { and } \quad k=k_{0},
$$

were observed as necessary and sufficient conditions for exponential decay of the system, and, in the general case, the system is polynomially stable. We remark that condition (1.5) is only mathematically sound and it is not given from physics.

In [2] the Bresse model for circular beams by adding two frictional dissipation in the system has been analyzed. The exponential stability was found if and only if $k=k_{0}$, with polynomial decay in the general case. The problem of optimality polynomial decay rate was also studied.

In [39] the stability of Bresse system has been explored. In that case, the two wave equations about the rotation angle and the longitudinal displacement are damped by two locally distributed feedbacks at the neighborhood of the boundary.

In [37] the exponential decay of a dissipative Bresse system has been showed by techniques developed in [33] and gave numerical simulations to support their results. 
When thermal effects are considered, the asymptotic behavior of the Bresse system may become more complicated because of the coupling between the elasticity and heat conduction. At present, there are some theoretical and numerical results on the asymptotic behavior of thermoelastic Bresse systems [24,25,33].

To our knowledge, this is the first paper where contact in the Bresse system has been performed. Moreover, exponential rate of decay is achieved without any restrictions on the parameters.

The first goal of the present paper is to obtain a global in time existence result for problem (1.1)-(1.3) by means of a Faedo-Galerkin scheme and suitable a priori estimates.

Secondly, we find the exponential stability by introducing a suitable Lyapunov functional and by using the multiplier method.

Next, fully discrete approximations are introduced by using a finite element method for the spatial approximation and the backward Euler scheme for the discretization of the time derivatives. Discrete stability results and a priori error estimates are obtained, from which the linear convergence is deduced under suitable regularity assumptions.

Finally, some numerical examples are shown to demonstrate the accuracy of the algorithm and the behavior of the solution.

\section{WELL-POSEDNESS}

Let $I:=(0, L)$. We introduce the following space

$$
H_{E}^{1}(I):=\left\{f \in H^{1}(I) ; f(0)=0\right\}
$$

and denote by $\|\cdot\|$ and $(\cdot, \cdot)$ the norm and the scalar product in $L^{2}(I)$, respectively. Before stating the main result of the existence and uniqueness of the solution of (1.1)-(1.3), we recall an inequality that will play a crucial role in all our calculation later.

Lemma 2.1. There exists $C>0$ such that, for all

$$
(\varphi, \psi, \omega) \in\left(H_{E}^{1}(I)\right)^{2} \times H_{0}^{1}(I),
$$

we have

$$
\left\|\varphi_{x}\right\|^{2}+\left\|\psi_{x}\right\|^{2}+\left\|\omega_{x}\right\|^{2} \leq C\left(\left\|\varphi_{x}+\psi+l \omega\right\|^{2}+\left\|\psi_{x}\right\|^{2}+\left\|\omega_{x}-l \varphi\right\|^{2}\right) .
$$

Proof. For the proof see Youssef [40].

Now, we enunciate our main theorem in this section.

Theorem 2.2. Assume that

$$
\varphi_{0} \in H_{E}^{1}(I) \cap H^{2}(I), \varphi_{1}, \psi_{0}, \psi_{1}, \omega_{0}, \omega_{1} \in H_{0}^{1}(I) \cap H^{2}(I),-g_{1} \leq \varphi_{0}(L) \leq g_{2},
$$

and

$$
\varphi_{0 x}=\varphi_{1 x}=\psi_{0 x}=\psi_{1 x}=\omega_{0 x}=\omega_{1 x}=0 \quad \text { for } \quad x=L .
$$

For each $T>0$, there exists a unique solution to contact problem (1.1)-(1.3) with the regularity:

$$
\begin{array}{ll}
\varphi, \varphi_{t}, \psi, \psi_{t} \in L^{\infty}\left(0, T ; H_{E}^{1}(I) \cap H^{2}(I)\right), & \varphi_{t t} \in L^{2}\left(0, T ; H_{E}^{1}(I)\right) \cap L^{\infty}\left(0, T ; L^{2}(I)\right), \\
\omega, \omega_{t} \in L^{\infty}\left(0, T ; H_{0}^{1}(I) \cap H^{2}(I)\right), & \psi_{t t}, \omega_{t t} \in L^{2}\left(0, T ; H_{0}^{1}(I)\right) \cap L^{\infty}\left(0, T ; L^{2}(I)\right), \\
\sigma \in L^{\infty}\left(0, T ; L^{2}(I)\right), & \sigma_{x} \in L^{2}\left(0, T ; L^{2}(I)\right) .
\end{array}
$$

Proof. For the proof, the Faedo-Galerkin method will be used. Indeed, several steps are required. 
Step 1. Applying integration by parts and using boundary conditions (1.2), the weak form associated to the continuous problem, obtained by multiplying equations (1.1) by test functions $\eta, \chi \in H_{E}^{1}(I)$ and $\xi \in H_{0}^{1}(I)$, is the following:

$$
\left.\begin{array}{c}
\rho_{1}\left(\varphi_{t t}, \eta\right)+k\left(\varphi_{x}+\psi+l \omega, \eta_{x}\right)+\zeta\left(\varphi_{x t}+\psi_{t}+l \omega_{t}, \eta_{x}\right)-k_{0} l\left(\omega_{x}-l \varphi, \eta\right)-\zeta l\left(\omega_{x t}-l \varphi_{t}, \eta\right) \\
\quad+\frac{1}{\varepsilon}\left(\left[\varphi(L, t)-g_{2}\right]_{+}-\left[-g_{1}-\varphi(L, t)\right]_{+}\right) \eta(L)=0, \\
\rho_{2}\left(\psi_{t t}, \chi\right)+b\left(\psi_{x}, \chi_{x}\right)+\zeta\left(\psi_{x t}, \chi_{x}\right)+k\left(\varphi_{x}+\psi+l \omega, \chi\right)+\zeta\left(\varphi_{x t}+\psi_{t}+l \omega_{t}, \chi\right)=0, \\
\rho_{1}\left(\omega_{t t}, \xi\right)+k_{0}\left(\omega_{x}-l \varphi, \xi_{x}\right)+\zeta\left(\omega_{x t}-l \varphi_{t}, \xi_{x}\right)+k l\left(\varphi_{x}+\psi+l \omega, \xi\right)+\zeta l\left(\varphi_{x t}+\psi_{t}+l \omega_{t}, \xi\right)=0 .
\end{array}\right\}
$$

For convenience, we look for approximate solutions of a modified version of (2.2) in which the initial data is zero. Let

$$
\widehat{\varphi}=\varphi-\varphi_{0}-t \varphi_{1}, \quad \widehat{\psi}=\psi-\psi_{0}-t \psi_{1}, \quad \widehat{\omega}=\omega-\omega_{0}-t \omega_{1},
$$

and choose $\left\{\eta_{i}\right\}_{i=1}^{\infty} \subset C^{\infty}(\bar{I})$ and $\left\{\mu_{i}\right\}_{i=1}^{\infty} \subset C^{\infty}(\bar{I})$ bases for $H_{E}^{1}(I)$ and $H_{0}^{1}(I)$, respectively. We introduce

$$
\varphi^{m}=\sum_{i=0}^{m} c_{i}(t) \eta_{i}(x), \quad \psi^{m}=\sum_{i=0}^{m} d_{i}(t) \eta_{i}(x), \quad \omega^{m}=\sum_{i=0}^{m} e_{i}(t) \mu_{i}(x),
$$

satisfying, $\forall \eta, \chi \in V^{m}:=\operatorname{span}\left\{\eta_{i}\right\}_{i=1}^{m}$ and $\forall \xi \in W^{m}:=\operatorname{span}\left\{\mu_{i}\right\}_{i=1}^{m}$, the variational equations

$$
\left.\begin{array}{l}
\rho_{1}\left(\varphi_{t t}^{m}, \eta\right)+k\left(\varphi_{x}^{m}+\psi^{m}+l \omega^{m}+\varphi_{0 x}+t \varphi_{1 x}+\psi_{0}+t \psi_{1}+l \omega_{0}+l t \omega_{1}, \eta_{x}\right) \\
\quad+\frac{1}{\varepsilon}\left(\left[\varphi^{m}(L, t)+\varphi_{0}(L)-g_{2}\right]_{+}-\left[-g_{1}-\varphi^{m}(L, t)-\varphi_{0}(L)\right]_{+}\right) \eta(L) \\
\quad-l k_{0}\left(w_{x}^{m}+\omega_{0 x}+t \omega_{1 x}-l \varphi^{m}-l \varphi_{0}-l t \varphi_{1}, \eta\right) \\
\quad+\zeta\left(\varphi_{x t}^{m}+\psi_{t}^{m}+l \omega_{t}^{m}+\varphi_{1 x}+\psi_{1}+l \omega_{1}, \eta_{x}\right) \\
\quad-\zeta l\left(w_{x t}^{m}+\omega_{1 x}-l \varphi_{t}^{m}-l \varphi_{1}, \eta\right)=0, \\
\rho_{2}\left(\psi_{t t}^{m}, \chi\right)+b\left(\psi_{x}^{m}+\psi_{0 x}+t \psi_{1 x}, \chi_{x}\right) \\
\quad+k\left(\varphi_{x}^{m}+\psi^{m}+l \omega^{m}+\varphi_{0 x}+t \varphi_{1 x}+\psi_{0}+t \psi_{1}+l \omega_{0}+l t \omega_{1}, \chi\right) \\
\quad+\zeta\left(\psi_{x t}^{m}+\psi_{1 x}, \chi_{x}\right)+\zeta\left(\varphi_{x t}^{m}+\psi_{t}^{m}+l w_{t}^{m}+\varphi_{1 x}+\psi_{1}+l \omega_{1}, \chi\right)=0, \\
\rho_{1}\left(\omega_{t t}^{m}, \xi\right)+k_{0}\left(\omega_{x}^{m}-l \varphi^{m}+\omega_{0 x}+t \omega_{1 x}-l \varphi_{0}-t l \varphi_{1}, \xi_{x}\right) \\
\quad+k l\left(\varphi_{x}^{m}+\psi^{m}+l \omega^{m}+\varphi_{0 x}+\psi_{0}+l \omega_{0}+t \varphi_{1 x}+t \psi_{1}+t l \omega_{1}, \xi\right) \\
\quad+\zeta\left(\omega_{x t}^{m}-l \varphi_{t}^{m}+\omega_{1 x}-l \varphi_{1 x}, \xi_{x}\right) \\
\quad+\zeta l\left(\varphi_{x t}^{m}+l \omega_{t}^{m}+\psi_{t}^{m}+\varphi_{1 x}+l \omega_{1}+\psi_{1}, \xi\right)=0,
\end{array}\right\}
$$

and the initial conditions $\varphi^{m}(\cdot, 0)=\psi^{m}(\cdot, 0)=\omega^{m}(\cdot, 0)=0$.

Substituting $\eta$ by $\varphi_{t}^{m}, \chi$ by $\psi_{t}^{m}, \xi$ by $\omega_{t}^{m}$ and adding the resulting variational equations, (2.3) gives

$$
\begin{aligned}
\frac{\rho_{1}}{2} \frac{\mathrm{d}}{\mathrm{d} t}\left\|\varphi_{t}^{m}\right\|^{2} & +\frac{\rho_{2}}{2} \frac{\mathrm{d}}{\mathrm{d} t}\left\|\psi_{t}^{m}\right\|^{2}+\frac{\rho_{1}}{2} \frac{\mathrm{d}}{\mathrm{d} t}\left\|\omega_{t}^{m}\right\|^{2}+\frac{k}{2} \frac{\mathrm{d}}{\mathrm{d} t}\left\|\varphi_{x}^{m}+\psi^{m}+l \omega^{m}\right\|^{2} \\
& +k\left(\varphi_{0 x}+t \varphi_{1 x}+\psi_{0 x}+t \psi_{1 x}+l \omega_{0 x}+l t \omega_{1 x}, \varphi_{x t}^{m}+\psi_{t}^{m}+l \omega_{t}^{m}\right) \\
& +\frac{k_{0}}{2} \frac{\mathrm{d}}{\mathrm{d} t}\left\|\omega_{x}^{m}-l \varphi^{m}\right\|^{2}+k_{0}\left(\omega_{0 x}+t \omega_{1 x}-l \varphi_{0}-l t \varphi_{1}, \omega_{x t}^{m}-l \varphi_{t}^{m}\right)
\end{aligned}
$$




$$
\begin{aligned}
& +\frac{b}{2} \frac{\mathrm{d}}{\mathrm{d} t}\left\|\psi_{x}^{m}\right\|^{2}+b\left(\psi_{0 x}+t \psi_{1 x}, \psi_{x t}^{m}\right) \\
& +\zeta\left\|\varphi_{x t}^{m}+\psi_{t}^{m}+l \omega_{t}^{m}\right\|^{2}+\zeta\left(\varphi_{1 x}+\psi_{1}+l \omega_{1}, \varphi_{x t}^{m}+\psi_{t}^{m}+l \omega_{t}^{m}\right) \\
& +\zeta\left\|\psi_{x t}^{m}\right\|^{2}+\zeta\left(\psi_{1 x}, \psi_{x t}^{m}\right)+\zeta\left\|\omega_{x t}^{m}-l \varphi_{t}^{m}\right\|^{2}+\zeta\left(\omega_{1 x}-l \varphi_{1}, \omega_{x t}^{m}-l \varphi_{t}^{m}\right) \\
& +\frac{1}{2 \varepsilon} \frac{\mathrm{d}}{\mathrm{d} t}\left(\left[\varphi^{m}(L, t)+\varphi_{0}(L)-g_{2}\right]_{+}^{2}+\left[-g_{1}-\varphi^{m}(L, t)-\varphi_{0}(L)\right]_{+}^{2}\right)=0 .
\end{aligned}
$$

Thus,

$$
\begin{aligned}
\frac{1}{2} \frac{\mathrm{d}}{\mathrm{d} t} & \left(\rho_{1}\left\|\varphi_{t}^{m}\right\|^{2}+\rho_{2}\left\|\psi_{t}^{m}\right\|^{2}+\rho_{1}\left\|\omega_{t}^{m}\right\|^{2}+k\left\|\varphi_{x}^{m}+\psi^{m}+l \omega^{m}\right\|^{2}+k_{0}\left\|\omega_{x}^{m}-l \varphi^{m}\right\|^{2}+b\left\|\psi_{x}^{m}\right\|^{2}\right. \\
& \left.+\frac{1}{\varepsilon}\left(\left[\varphi^{m}(L, t)+\varphi_{0}(L)-g_{2}\right]_{+}^{2}+\left[-g_{1}-\varphi^{m}(L, t)-\varphi_{0}(L)\right]_{+}^{2}\right)\right) \\
& +\zeta\left\|\varphi_{x t}^{m}+\psi_{t}^{m}+l \omega_{t}^{m}\right\|^{2}+\zeta\left\|\psi_{x t}^{m}\right\|^{2}+\zeta\left\|\omega_{x t}^{m}-l \varphi_{t}^{m}\right\|^{2} \\
= & -k\left(\varphi_{0 x}+t \varphi_{1 x}+\psi_{0 x}+t \psi_{1 x}+l \omega_{0 x}+l t \omega_{1 x}, \varphi_{x t}^{m}+\psi_{t}^{m}+l \omega_{t}^{m}\right) \\
& -k_{0}\left(\omega_{0 x}+t \omega_{1 x}-l \varphi_{0}-l t \varphi_{1}, \omega_{x t}^{m}-l \varphi_{t}^{m}\right)-b\left(\psi_{0 x}+t \psi_{1 x}, \psi_{x t}^{m}\right) \\
& -\zeta\left(\varphi_{1 x}+\psi_{1}+l \omega_{1}, \varphi_{x t}^{m}+\psi_{t}^{m}+l \omega_{t}^{m}\right)-\zeta\left(\psi_{1 x}, \psi_{x t}^{m}\right)-\zeta\left(\omega_{1 x}-l \varphi_{1}, \omega_{x t}^{m}-l \varphi_{t}^{m}\right) .
\end{aligned}
$$

Using Young's inequality, for all non-negative constants $\varepsilon_{1}, \varepsilon_{2}, \varepsilon_{3}, \varepsilon_{4}, \varepsilon_{5}$ and $\varepsilon_{6}$, we obtain from (2.4)

$$
\begin{aligned}
\frac{1}{2} \frac{\mathrm{d}}{\mathrm{d} t} & \left(\rho_{1}\left\|\varphi_{t}^{m}\right\|^{2}+\rho_{2}\left\|\psi_{t}^{m}\right\|^{2}+\rho_{1}\left\|\omega_{t}^{m}\right\|^{2}+k\left\|\varphi_{x}^{m}+\psi^{m}+l \omega^{m}\right\|^{2}+k_{0}\left\|\omega_{x}^{m}-l \varphi^{m}\right\|^{2}+b\left\|\psi_{x}^{m}\right\|^{2}\right. \\
& \left.+\frac{1}{\varepsilon}\left(\left[\varphi^{m}(L, t)+\varphi_{0}(L)-g_{2}\right]_{+}^{2}+\left[-g_{1}-\varphi^{m}(L, t)-\varphi_{0}(L)\right]_{+}^{2}\right)\right) \\
& +\zeta\left\|\varphi_{x t}^{m}+\psi_{t}^{m}+l \omega_{t}^{m}\right\|^{2}+\zeta\left\|\psi_{x t}^{m}\right\|^{2}+\zeta\left\|\omega_{x t}^{m}-l \varphi_{t}^{m}\right\|^{2} \\
\leq & C+\frac{k}{2} \varepsilon_{1}\left\|\varphi_{x t}^{m}+\psi_{t}^{m}+l \omega_{t}^{m}\right\|^{2}+\frac{k_{0}}{2} \varepsilon_{2}\left\|\omega_{x t}^{m}-l \varphi_{t}^{m}\right\|^{2}+\frac{b}{2} \varepsilon_{3}\left\|\psi_{x t}^{m}\right\|^{2} \\
& +\zeta \varepsilon_{4}\left\|\varphi_{x t}^{m}+\psi_{t}^{m}+l \omega_{t}^{m}\right\|^{2}+\zeta \varepsilon_{5}\left\|\psi_{x t}^{m}\right\|^{2}+\zeta \varepsilon_{6}\left\|\omega_{x t}^{m}-l \varphi_{t}^{m}\right\|^{2},
\end{aligned}
$$

where $C$ is a positive constant that depends on $\varphi_{i}, \varphi_{i x}, \psi_{i}, \psi_{i x}, \omega_{i}, \omega_{i x}$, for $i=0,1$.

Choosing $\varepsilon_{1}=\frac{\zeta}{k}, \varepsilon_{2}=\frac{\zeta}{k_{0}}, \varepsilon_{3}=\frac{\zeta}{b}, \varepsilon_{4}=\varepsilon_{5}=\varepsilon_{6}=\frac{1}{4}$, (2.5) leads to

$$
\begin{aligned}
\frac{1}{2} \frac{\mathrm{d}}{\mathrm{d} t}( & \rho_{1}\left\|\varphi_{t}^{m}\right\|^{2}+\rho_{2}\left\|\psi_{t}^{m}\right\|^{2}+\rho_{1}\left\|\omega_{t}^{m}\right\|^{2}+k\left\|\varphi_{x}^{m}+\psi^{m}+l \omega^{m}\right\|^{2}+k_{0}\left\|\omega_{x}^{m}-l \varphi^{m}\right\|^{2}+b\left\|\psi_{x}^{m}\right\|^{2} \\
& \left.+\frac{1}{\varepsilon}\left(\left[\varphi^{m}(L, t)+\varphi_{0}(L)-g_{2}\right]_{+}^{2}+\left[-g_{1}-\varphi^{m}(L, t)-\varphi_{0}(L)\right]_{+}^{2}\right)\right) \\
& +\frac{\zeta}{4}\left\|\varphi_{x t}^{m}+\psi_{t}^{m}+\omega_{t}^{m}\right\|^{2}+\frac{\zeta}{4}\left\|\psi_{x}^{m}\right\|^{2}+\frac{\zeta}{4}\left\|\omega_{x t}^{m}-l \varphi_{t}^{m}\right\|^{2} \leq C .
\end{aligned}
$$

Thereby,

$$
\begin{aligned}
\rho_{1}\left\|\varphi_{t}^{m}(\cdot, T)\right\|^{2} & +\rho_{2}\left\|\psi_{t}^{m}(\cdot, T)\right\|^{2}+\rho_{1}\left\|\omega_{t}^{m}(\cdot, T)\right\|^{2}+k\left\|\varphi_{x}^{m}(\cdot, T)+\psi^{m}(\cdot, T)+l \omega^{m}(\cdot, T)\right\|^{2}+k_{0} \| \omega_{x}^{m}(\cdot, T) \\
& -l \varphi^{m}(\cdot, T)\left\|^{2}+b\right\| \psi_{x}^{m}(\cdot, T) \|^{2}+\frac{1}{\varepsilon}\left(\left[\varphi^{m}(L, T)+\varphi_{0}(L)-g_{2}\right]_{+}^{2}+\left[-g_{1}-\varphi^{m}(L, T)-\varphi_{0}(L)\right]_{+}^{2}\right) \\
& +\frac{\zeta}{2} \int_{0}^{T}\left(\left\|\varphi_{x t}^{m}+\psi_{t}^{m}+\omega_{t}^{m}\right\|^{2}+\left\|\psi_{x t}^{m}\right\|^{2}+\left\|\omega_{x t}^{m}-l \varphi_{t}^{m}\right\|^{2}\right) \mathrm{d} t \leq C
\end{aligned}
$$


Step 2. Differentiating (2.3) with respect to $t$ and substituting $\eta$ by $\varphi_{t t}^{m}, \chi$ by $\psi_{t t}^{m}$, and $\xi$ by $\omega_{t t}^{m}$, we get

$$
\begin{aligned}
\frac{1}{2} \frac{\mathrm{d}}{\mathrm{d} t}( & \left.\rho_{1}\left\|\varphi_{t t}^{m}\right\|^{2}+\rho_{2}\left\|\psi_{t t}^{m}\right\|^{2}+\rho_{1}\left\|\omega_{t t}^{m}\right\|^{2}+k\left\|\varphi_{x t}^{m}+\psi_{t}^{m}+l \omega_{t}^{m}\right\|^{2}+k_{0}\left\|\omega_{x t}^{m}-l \varphi_{t}^{m}\right\|^{2}+b\left\|\psi_{x t}^{m}\right\|^{2}\right) \\
& +\zeta\left\|\varphi_{x t t}^{m}+\psi_{t t}^{m}+l \omega_{t t}^{m}\right\|^{2}+\zeta\left\|\psi_{x t t}^{m}\right\|^{2}+\zeta l\left\|\omega_{x t t}^{m}-l \varphi_{t t}^{m}\right\|^{2} \\
= & \left.-\frac{1}{\varepsilon} \frac{\mathrm{d}}{\mathrm{d} t}\left(\varphi^{m}(L, t)+\varphi_{0}(L)-g_{2}\right]_{+}-\left[-g_{1}-\varphi_{0}(L)-\varphi^{m}(L, t)\right]_{+}\right) \varphi_{t t}^{m}(L, t) \\
& -k\left(\varphi_{1 x}+\psi_{1}+l \omega_{1}, \varphi_{x t t}^{m}+\psi_{t t}^{m}+l \omega_{t t}^{m}\right)-b\left(\psi_{1 x}, \psi_{x t t}^{m}\right)-k_{0}\left(\omega_{1 x}-l \varphi_{1}, \omega_{x t t}^{m}-l \varphi_{t t}^{m}\right) .
\end{aligned}
$$

Next, using Young's inequality, we obtain

$$
\begin{aligned}
\frac{1}{2} \frac{\mathrm{d}}{\mathrm{d} t} & \left(\rho_{1}\left\|\varphi_{t t}^{m}\right\|^{2}+\rho_{2}\left\|\psi_{t t}^{m}\right\|^{2}+\rho_{1}\left\|\omega_{t t}^{m}\right\|^{2}+k\left\|\varphi_{x t}^{m}+\psi_{t}^{m}+l \omega_{t}^{m}\right\|^{2}+k_{0}\left\|\omega_{x t}^{m}-l \varphi_{t}^{m}\right\|^{2}+b\left\|\psi_{x t}^{m}\right\|^{2}\right) \\
& +\zeta\left\|\varphi_{x t t}^{m}+\psi_{t t}^{m}+l \omega_{t t}^{m}\right\|^{2}+\zeta\left\|\psi_{x t t}^{m}\right\|^{2}+\zeta l\left\|\omega_{x t t}^{m}-l \varphi_{t t}^{m}\right\|^{2} \\
& \leq \frac{\delta_{1}}{\varepsilon}\left(\varphi_{t}^{m}(L, t)\right)^{2}+\frac{1}{\varepsilon \delta_{1}}\left(\varphi_{t t}^{m}(L, t)\right)^{2}+k \delta_{2}\left\|\varphi_{x t t}^{m}+\psi_{t t}^{m}+l \omega_{t t}^{m}\right\|^{2}+b \delta_{3}\left\|\psi_{x t t}^{m}\right\|^{2}+k_{0} \delta_{4}\left\|\omega_{x t t}^{m}-l \varphi_{t t}^{m}\right\|^{2}+C_{1},
\end{aligned}
$$

where $C_{1}$ depends on $\delta_{2}, \delta_{3}, \delta_{4}, k, b, l, \varphi_{1}, \varphi_{1 x}, \psi_{1}, \omega_{1}, \omega_{1 x}$, and $\psi_{1 x}$.

On the other hand, we have

$$
\left(\varphi_{t}^{m}(L, t)\right)^{2} \leq L\left\|\varphi_{x t}^{m}\right\|^{2} \leq c\left(\left\|\varphi_{x t}^{m}+\psi_{t}^{m}+l \omega_{t}^{m}\right\|^{2}+\left\|\psi_{t}^{m}\right\|^{2}+\left\|\omega_{t}^{m}\right\|^{2}\right)
$$

and

$$
\left(\varphi_{t t}^{m}(L, t)\right)^{2} \leq L\left\|\varphi_{x t t}^{m}\right\|^{2} \leq c\left(\left\|\varphi_{x t t}^{m}+\psi_{t t}^{m}+l \omega_{t t}^{m}\right\|^{2}+\left\|\psi_{t t}^{m}\right\|^{2}+\left\|\omega_{t t}^{m}\right\|^{2}\right) .
$$

Therefore, inserting (2.8) and (2.9) into (2.7) it leads to

$$
\begin{aligned}
\frac{1}{2} \frac{\mathrm{d}}{\mathrm{d} t} & \left(\rho_{1}\left\|\varphi_{t t}^{m}\right\|^{2}+\rho_{2}\left\|\psi_{t t}^{m}\right\|^{2}+\rho_{1}\left\|\omega_{t t}^{m}\right\|^{2}+k\left\|\varphi_{x t}^{m}+\psi_{t}^{m}+l \omega_{t}^{m}\right\|^{2}+k_{0}\left\|\omega_{x t}^{m}-l \varphi_{t}^{m}\right\|^{2}+b\left\|\psi_{x t}^{m}\right\|^{2}\right) \\
& +\zeta\left\|\varphi_{x t t}^{m}+\psi_{t t}^{m}+l \omega_{t t}^{m}\right\|^{2}+\zeta\left\|\psi_{x t t}^{m}\right\|^{2}+\zeta l\left\|\omega_{x t t}^{m}-l \varphi_{t t}^{m}\right\|^{2} \\
\leq & \frac{c \delta_{1}}{\varepsilon}\left(\left\|\varphi_{x t}^{m}+\psi_{t}^{m}+l \omega_{t}^{m}\right\|^{2}+\left\|\psi_{t}^{m}\right\|^{2}+\left\|\omega_{t}^{m}\right\|^{2}\right)+\frac{c}{\varepsilon \delta_{1}}\left(\left\|\varphi_{x t t}^{m}+\psi_{t t}^{m}+l \omega_{t t}^{m}\right\|^{2}+\left\|\psi_{t t}^{m}\right\|^{2}+\left\|\omega_{t t}^{m}\right\|^{2}\right) \\
& \quad+k \delta_{2}\left\|\varphi_{x t t}^{m}+\psi_{t t}^{m}+l \omega_{t t}^{m}\right\|^{2}+b \delta_{3}\left\|\psi_{x t t}^{m}\right\|^{2}+k_{0} \delta_{4}\left\|\omega_{x t t}^{m}-l \varphi_{t t}^{m}\right\|^{2}+C_{1} .
\end{aligned}
$$

However, the terms $\left\|\psi_{t}^{m}\right\|$ and $\left\|\omega_{t}^{m}\right\|$ are bounded due to (2.6). Thus, (2.10) implies

$$
\begin{aligned}
\frac{1}{2} \frac{\mathrm{d}}{\mathrm{d} t} & \left(\rho_{1}\left\|\varphi_{t t}^{m}\right\|^{2}+\rho_{2}\left\|\psi_{t t}^{m}\right\|^{2}+\rho_{1}\left\|\omega_{t t}^{m}\right\|^{2}+k\left\|\varphi_{x t}^{m}+\psi_{t}^{m}+l \omega_{t}^{m}\right\|^{2}+k_{0}\left\|\omega_{x t}^{m}-l \varphi_{t}^{m}\right\|^{2}+b\left\|\psi_{x t}^{m}\right\|^{2}\right) \\
& +\zeta\left\|\varphi_{x t t}^{m}+\psi_{t t}^{m}+l \omega_{t t}^{m}\right\|^{2}+\zeta\left\|\psi_{x t t}^{m}\right\|^{2}+\zeta l\left\|\omega_{x t t}^{m}-l \varphi_{t t}^{m}\right\|^{2} \\
\leq & \left(\frac{c}{\varepsilon \delta_{1}}+k \delta_{2}\right)\left\|\varphi_{x t t}^{m}+\psi_{t t}^{m}+l \omega_{t t}^{m}\right\|^{2}+b \delta_{3}\left\|\psi_{x t t}^{m}\right\|^{2}+\frac{c}{\varepsilon \delta_{1}}\left\|\psi_{t t}^{m}\right\|^{2} \\
& +\frac{c}{\varepsilon \delta_{1}}\left\|\omega_{t t}^{m}\right\|^{2}+\frac{c \delta_{1}}{\varepsilon}\left\|\varphi_{x t}^{m}+\psi_{t}^{m}+l \omega_{t}^{m}\right\|^{2}+k_{0} \delta_{4}\left\|\omega_{x t t}^{m}-l \varphi_{t t}^{m}\right\|^{2}+C_{3} .
\end{aligned}
$$

Then, using (2.6) and selecting $\delta_{1}, \delta_{2}, \delta_{3}$, and $\delta_{4}$ such that

$$
\zeta-\left(\frac{c}{\varepsilon \delta_{1}}+k \delta_{2}\right)>\frac{\zeta}{2}, \quad \zeta-b \delta_{3}>\frac{\zeta}{2} \quad \text { and } \quad \zeta l-k_{0} \delta_{4}>\frac{\zeta l}{2}
$$


estimate (2.11) gives

$$
\begin{aligned}
& \frac{1}{2} \frac{\mathrm{d}}{\mathrm{d} t}\left(\rho_{1}\left\|\varphi_{t t}^{m}\right\|^{2}+\rho_{2}\left\|\psi_{t t}^{m}\right\|^{2}+\rho_{1}\left\|\omega_{t t}^{m}\right\|^{2}+k\left\|\varphi_{x t}^{m}+\psi_{t}^{m}+l \omega_{t}^{m}\right\|^{2}+k_{0}\left\|\omega_{x t}^{m}-l \varphi_{t}^{m}\right\|^{2}+b\left\|\psi_{x t}^{m}\right\|^{2}\right) \\
& \quad+\frac{\zeta}{2}\left\|\varphi_{x t t}^{m}+\psi_{t t}^{m}+l \omega_{t t}^{m}\right\|^{2}+\frac{\zeta}{2}\left\|\psi_{x t t}^{m}\right\|^{2}+\frac{\zeta l}{2}\left\|\omega_{x t t}^{m}-l \varphi_{t t}^{m}\right\|^{2} \\
& \leq \frac{c}{\varepsilon \delta_{1}}\left\|\psi_{t t}^{m}\right\|^{2}+\frac{c}{\varepsilon \delta_{1}}\left\|\omega_{t t}^{m}\right\|^{2}+C_{3} .
\end{aligned}
$$

Now, integrating over $[0, T]$, we obtain

$$
\begin{aligned}
\frac{\rho_{1}}{2}\left\|\varphi_{t t}^{m}(\cdot, T)\right\|^{2} & +\frac{\rho_{2}}{2}\left\|\psi_{t t}^{m}(\cdot, T)\right\|^{2}+\frac{\rho_{1}}{2}\left\|\omega_{t t}^{m}(\cdot, T)\right\|^{2}+\frac{k}{2}\left\|\varphi_{x t}^{m}(\cdot, T)+\psi_{t}^{m}(\cdot, T)+l \omega_{t}^{m}(\cdot, T)\right\|^{2} \\
& +\frac{k_{0}}{2}\left\|\omega_{x t}^{m}(\cdot, T)-l \varphi_{t}^{m}(\cdot, T)\right\|^{2}+\frac{b}{2}\left\|\psi_{x t}^{m}(\cdot, T)\right\|^{2}+\frac{\zeta}{2} \int_{0}^{T}\left\|\varphi_{x t t}^{m}+\psi_{t t}^{m}+l \omega_{t t}^{m}\right\|^{2} \mathrm{~d} t \\
& +\frac{\zeta}{2} \int_{0}^{T}\left\|\psi_{x t t}^{m}\right\|^{2} \mathrm{~d} t+\frac{\zeta l}{2} \int_{0}^{T}\left\|\omega_{x t t}^{m}-l \varphi_{t t}^{m}\right\|^{2} \mathrm{~d} t \\
\leq & \frac{\rho_{1}}{2}\left\|\varphi_{t t}^{m}(\cdot, 0)\right\|^{2}+\frac{\rho_{2}}{2}\left\|\psi_{t t}^{m}(\cdot, 0)\right\|^{2}+\frac{\rho_{1}}{2}\left\|\omega_{t t}^{m}(\cdot, 0)\right\|^{2}+\frac{c}{\varepsilon \delta_{1}} \int_{0}^{T}\left\|\psi_{t t}^{m}\right\|^{2} \mathrm{~d} t \\
& +\frac{c}{\varepsilon \delta_{1}} \int_{0}^{T}\left\|\omega_{t t}^{m}\right\|^{2} \mathrm{~d} t+T C_{3} .
\end{aligned}
$$

Due to (2.6), (2.12) implies that

$$
\begin{aligned}
\frac{\rho_{1}}{2}\left\|\varphi_{t t}^{m}(\cdot, T)\right\|^{2} & +\frac{\rho_{2}}{2}\left\|\psi_{t t}^{m}(\cdot, T)\right\|^{2}+\frac{\rho_{1}}{2}\left\|\omega_{t t}^{m}(\cdot, T)\right\|^{2}+\frac{k}{2}\left\|\varphi_{x t}^{m}(\cdot, T)+\psi_{t}^{m}(\cdot, T)+l \omega_{t}^{m}(\cdot, T)\right\|^{2} \\
& +\frac{k_{0}}{2}\left\|\omega_{x t}^{m}(\cdot, T)-l \varphi_{t}^{m}(\cdot, T)\right\|^{2}+\frac{b}{2}\left\|\psi_{x t}^{m}(\cdot, T)\right\|^{2}+\frac{\zeta}{2} \int_{0}^{T}\left\|\varphi_{x t t}^{m}+\psi_{t t}^{m}+l \omega_{t t}^{m}\right\|^{2} \mathrm{~d} t \\
& +\frac{\zeta}{2} \int_{0}^{T}\left\|\psi_{x t t}^{m}\right\|^{2} \mathrm{~d} t+\frac{\zeta l}{2} \int_{0}^{T}\left\|\omega_{x t t}^{m}-l \varphi_{t t}^{m}\right\|^{2} \mathrm{~d} t \\
\leq & \frac{\rho_{1}}{2}\left\|\varphi_{t t}^{m}(\cdot, 0)\right\|^{2}+\frac{\rho_{2}}{2}\left\|\psi_{t t}^{m}(\cdot, 0)\right\|^{2}+\frac{\rho_{1}}{2}\left\|\omega_{t t}^{m}(\cdot, 0)\right\|^{2}+\frac{c}{\varepsilon \delta_{1}} \int_{0}^{T}\left\|\psi_{t t}^{m}\right\|^{2} \mathrm{~d} t \\
& +\frac{c}{\varepsilon \delta_{1}} \int_{0}^{T}\left\|\omega_{t t}^{m}\right\|^{2} \mathrm{~d} t+\underbrace{\frac{2 C}{\zeta}+T C_{3}}_{:=C_{4}} .
\end{aligned}
$$

Now, let us prove that $\left\|\varphi_{t t}^{m}(\cdot, 0)\right\|,\left\|\psi_{t t}^{m}(\cdot, 0)\right\|$, and $\left\|\omega_{t t}^{m}(\cdot, 0)\right\|$ are bounded. Taking $t=0$ in $(2.3)$, substituting $\eta$ by $\varphi_{t t}^{m}(\cdot, 0)$ and integrating by parts, we obtain

$$
\begin{aligned}
\rho_{1}\left\|\varphi_{t t}^{m}(\cdot, 0)\right\|^{2} \leq & k\left\|\varphi_{0 x x}+\psi_{0 x}+l \omega_{0 x}\right\|\left\|\varphi_{t t}^{m}(\cdot, 0)\right\|+\zeta\left\|\varphi_{1 x x}+\psi_{1 x}+l \omega_{1 x}\right\|\left\|\varphi_{t t}^{m}(\cdot, 0)\right\| \\
& +l k_{0}\left\|w_{0 x}-l \varphi_{0}\right\|\left\|\varphi_{t t}^{m}(\cdot, 0)\right\|+\zeta l\left\|w_{1 x}-l \varphi_{1}\right\|\left\|\varphi_{t t}^{m}(\cdot, 0)\right\| .
\end{aligned}
$$

Next, using the following Young inequality

$$
a b \leq C_{\gamma} a^{2}+\gamma b^{2}, \quad \forall \gamma>0,
$$

we get, for $\gamma_{1}, \gamma_{2}, \gamma_{3}$, and $\gamma_{4}$ small enough,

$$
\rho_{1}\left\|\varphi_{t t}^{m}(\cdot, 0)\right\|^{2} \leq k C_{\gamma_{1}}\left\|\varphi_{0 x x}+\psi_{0 x}+l \omega_{0 x}\right\|+\zeta C_{\gamma_{2}}\left\|\varphi_{1 x x}+\psi_{1 x}+l \omega_{1 x}\right\|
$$




$$
+l k_{0} C_{\gamma_{3}}\left\|w_{0 x}-l \varphi_{0}\right\|+\zeta l C_{\gamma_{4}}\left\|w_{1 x}-l \varphi_{1}\right\|
$$

Therefore, $\left\|\varphi_{t t}^{m}(\cdot, 0)\right\|$ is bounded because $\varphi_{0}, \varphi_{1}, \psi_{0}, \psi_{1}, \omega_{0}, \omega_{1} \in H^{2}(I)$. By repeating the same arguments, we deduce that $\left\|\psi_{t t}^{m}(\cdot, 0)\right\|$ and $\left\|\omega_{t t}^{m}(\cdot, 0)\right\|$ are bounded.

Consequently, applying Gronwall's inequality to (2.13) it results that

$$
\begin{aligned}
\frac{\rho_{1}}{2}\left\|\varphi_{t t}^{m}(\cdot, T)\right\|^{2} & +\frac{\rho_{2}}{2}\left\|\psi_{t t}^{m}(\cdot, T)\right\|^{2}+\frac{\rho_{1}}{2}\left\|\omega_{t t}^{m}(\cdot, T)\right\|^{2}+\frac{k}{2}\left\|\varphi_{x t}^{m}(\cdot, T)+\psi_{t}^{m}(\cdot, T)+l \omega_{t}^{m}(\cdot, T)\right\|^{2} \\
& +\frac{k_{0}}{2}\left\|\omega_{x t}^{m}(\cdot, T)-l \varphi_{t}^{m}(\cdot, T)\right\|^{2}+\frac{b}{2}\left\|\psi_{x t}^{m}(\cdot, T)\right\|^{2}+\frac{\zeta}{2} \int_{0}^{T}\left\|\varphi_{x t t}^{m}+\psi_{t t}^{m}+l \omega_{t t}^{m}\right\|^{2} \mathrm{~d} t \\
& +\frac{\zeta}{2} \int_{0}^{T}\left\|\psi_{x t t}^{m}\right\|^{2} \mathrm{~d} t+\frac{\zeta l}{2} \int_{0}^{T}\left\|\omega_{x t t}^{m}-l \varphi_{t t}^{m}\right\|^{2} \mathrm{~d} t \leq C_{3} .
\end{aligned}
$$

From the above estimates and using (2.1), there exist subsequences denoted also by $\left\{\varphi^{m}\right\},\left\{\psi^{m}\right\}$, and $\left\{\omega^{m}\right\}$ such that:

$$
\begin{aligned}
& \varphi_{t}^{m} \stackrel{*}{\rightarrow} \widehat{\varphi}_{t}, \quad \psi_{t}^{m} \stackrel{*}{\rightarrow} \widehat{\psi}_{t}, \quad \omega_{t}^{m} \stackrel{*}{\rightarrow} \widehat{\omega}_{t} \quad \text { in } \quad L^{\infty}\left(0, T ; H_{E}^{1}(I)\right), \\
& \varphi_{t}^{m} \rightarrow \widehat{\varphi}_{t}, \quad \psi_{t}^{m} \rightarrow \widehat{\psi}_{t}, \quad \omega_{t}^{m} \rightarrow \widehat{\omega}_{t} \quad \text { in } \quad L^{2}\left(0, T ; H_{E}^{1}(I)\right), \\
& \varphi^{m} \stackrel{*}{\rightarrow} \widehat{\varphi}, \quad \psi^{m} \stackrel{*}{\rightarrow} \widehat{\psi}, \quad \omega^{m} \stackrel{*}{\rightarrow} \widehat{\omega} \quad \text { in } \quad L^{\infty}\left(0, T ; H_{E}^{1}(I)\right), \\
& \varphi_{t t}^{m} \stackrel{*}{\rightarrow} \widehat{\varphi}_{t t}, \quad \psi_{t t}^{m} \stackrel{*}{\rightarrow} \widehat{\psi}_{t t}, \quad \omega_{t t}^{m} \stackrel{*}{\rightarrow} \widehat{\omega}_{t t} \quad \text { in } \quad L^{\infty}\left(0, T ; L^{2}(I)\right), \\
& \varphi_{t t}^{m} \rightarrow \widehat{\varphi}_{t t}, \quad \psi_{t t}^{m} \rightarrow \widehat{\psi}_{t t}, \quad \omega_{t t}^{m} \rightarrow \widehat{\omega}_{t t} \quad \text { in } \quad L^{2}\left(0, T ; H_{E}^{1}(I)\right), \\
& \frac{1}{\varepsilon}\left[\varphi^{m}(L, t)+\varphi_{0}(L)-g_{2}\right] \stackrel{*}{\rightarrow} \frac{1}{\varepsilon}\left[\widehat{\varphi}(L, t)+\varphi_{0}(L)-g_{2}\right] \quad \text { in } \quad L^{\infty}(0, T), \\
& \frac{1}{\varepsilon}\left[-g_{1}-\varphi^{m}(L, t)-\varphi_{0}(L)\right] \stackrel{*}{\rightarrow} \frac{1}{\varepsilon}\left[-g_{1}-\widehat{\varphi}(L, t)-\varphi_{0}(L)\right] \quad \text { in } \quad L^{\infty}(0, T) \text {. }
\end{aligned}
$$

Moreover, the fact that $\left\{\varphi^{m}(L, t)\right\}$ and $\left\{\varphi_{t}^{m}(L, t)\right\}$ are bounded implies that

$$
\varphi^{m}(L, t) \rightarrow \widehat{\varphi}(L, t) \quad \text { in } H^{1}(0, T) .
$$

Therefore, the compactness of $H^{1}(0, T) \subset L^{2}(0, T)$ leads to

$$
\varphi^{m}(L, t) \rightarrow \widehat{\varphi}(L, t) \quad \text { in } L^{2}(0, T) .
$$

Next, we have

$$
\left\|\left[\varphi^{m}(L, t)+\varphi_{0}(L)-g_{2}\right]_{+}-\left[\widehat{\varphi}(L, t)+\varphi_{0}(L)-g_{2}\right]_{+}\right\|_{L^{2}(0, T)}^{2} \leq\left\|\varphi^{m}(L, t)-\widehat{\varphi}(L, t)\right\|_{L^{2}(0, T)} .
$$

Consequently,

$$
\left[\varphi^{m}(L, t)+\varphi_{0}(L)-g_{2}\right]_{+} \rightarrow\left[\widehat{\varphi}(L, t)+\varphi_{0}(L)-g_{2}\right]_{+} \quad \text { in } \quad L^{2}(0, T) .
$$

Similarly, we show that

$$
\left[-g_{1}-\varphi^{m}(L, t)-\varphi_{0}(L)\right]_{+} \rightarrow\left[-g_{1}-\widehat{\varphi}(L, t)-\varphi_{0}(L)\right]_{+} \quad \text { in } \quad L^{2}(0, T) .
$$

Taking the limit in (2.3) as $m \rightarrow+\infty$ and reversing the change of variables, we deduce that $\varphi, \psi$ and $\omega$ satisfy variational problem (2.2). By standard arguments, the existence result follows.

Step 3. In this step, we shall prove the uniqueness of the solution. So, let us suppose that $\left(\varphi_{1}, \psi_{1}, \omega_{1}\right)$ and $\left(\varphi_{2}, \psi_{2}, \omega_{2}\right)$ are two solutions to equations (1.1) and let

$$
\varphi=\varphi_{1}-\varphi_{2}, \quad \psi=\psi_{1}-\psi_{2}, \quad \omega=\omega_{1}-\omega_{2} .
$$


Thus, from the weak formulation (2.2) we get

$$
\begin{aligned}
\frac{1}{2} \frac{\mathrm{d}}{\mathrm{d} t} & \left(\rho_{1}\left\|\varphi_{t}\right\|^{2}+\rho_{2}\left\|\psi_{t}\right\|^{2}+\rho_{1}\left\|\omega_{t}\right\|^{2}+k\left\|\varphi_{x}+\psi+l \omega\right\|^{2}+k_{0}\left\|\omega_{x}-l \varphi\right\|^{2}+b\left\|\psi_{x}\right\|^{2}\right) \\
& +\zeta\left\|\varphi_{x t}+\psi_{t}+l \omega_{t}\right\|^{2}+\zeta\left\|\omega_{x t}-l \varphi_{t}\right\|^{2}+\zeta\left\|\psi_{x t}\right\|^{2} \\
& +\frac{1}{\varepsilon}\left(\left[\varphi_{1}(L, t)+\varphi_{0}(L)-g_{2}\right]_{+}-\left[-g_{1}-\varphi_{1}(L, t)-\varphi_{0}(L)\right]_{+}\right) \varphi_{t}(L, t) \\
& -\frac{1}{\varepsilon}\left(\left[\varphi_{2}(L, t)+\varphi_{0}(L)-g_{2}\right]_{+}+\left[-g_{1}-\varphi_{2}(L, t)-\varphi_{0}(L)\right]_{+}\right) \varphi_{t}(L, t)=0 .
\end{aligned}
$$

Next, let us estimate the two last term $I_{1}-I_{2}$ in (2.17). First, note that

$$
\varphi_{1}(L, t)=\int_{0}^{L} \varphi_{1 x}(t, x) \mathrm{d} x \quad \text { and } \quad \varphi_{2}(L, t)=\int_{0}^{L} \varphi_{2 x}(t, x) \mathrm{d} x .
$$

Applying the Young inequality, for all $\delta>0$, we get

$$
\begin{aligned}
& \frac{1}{\varepsilon}( {\left.\left[\varphi_{1}(L, t)+\varphi_{0}(L)-g_{2}\right]_{+}-\left[-g_{1}-\varphi_{1}(L, t)-\varphi_{0}(L)\right]_{+}\right) \varphi_{t}(L, t) } \\
&-\frac{1}{\varepsilon}\left(\left[\varphi_{2}(L, t)+\varphi_{0}(L)-g_{2}\right]_{+}+\left[-g_{1}-\varphi_{2}(L, t)-\varphi_{0}(L)\right]_{+}\right) \varphi_{t}(L, t) \\
& \leq \frac{\delta}{2 \varepsilon}\left|\varphi_{t}(L, t)\right|^{2} \\
& \quad+\frac{1}{2 \delta \varepsilon}\left(\left[\varphi_{1}(L, t)+\varphi_{0}(L)-g_{2}\right]_{+}-\left[-g_{1}-\varphi_{1}(L, t)-\varphi_{0}(L)\right]_{+}\right. \\
&\left.\quad-\left[\varphi_{2}(L, t)+\varphi_{0}(L)-g_{2}\right]_{+}+\left[-g_{1}-\varphi_{2}(L, t)-\varphi_{0}(L)\right]_{+}\right)^{2}
\end{aligned}
$$

On the other hand, we have

$$
\left|\left[\varphi_{1}(L, t)+\varphi_{0}(L)-g_{2}\right]_{+}-\left[\varphi_{2}(L, t)+\varphi_{0}(L)-g_{2}\right]_{+}\right| \leq\left|\varphi_{1}(L, t)-\varphi_{2}(L, t)\right|
$$

and

$$
\left|-\left[-g_{1}-\varphi_{1}(L, t)-\varphi_{0}(L)\right]_{+}+\left[-g_{1}-\varphi_{2}(L, t)-\varphi_{0}(L)\right]_{+}\right| \leq\left|\varphi_{1}(L, t)-\varphi_{2}(L, t)\right| .
$$

Hence, using (2.19) and (2.20) in (2.18), we obtain

$$
\begin{aligned}
\frac{1}{\varepsilon}( & {\left.\left[\varphi_{1}(L, t)+\varphi_{0}(L)-g_{2}\right]_{+}-\left[-g_{1}-\varphi_{1}(L, t)-\varphi_{0}(L)\right]_{+}\right) \varphi_{t}(L, t) } \\
& -\frac{1}{\varepsilon}\left(\left[\varphi_{2}(L, t)+\varphi_{0}(L)-g_{2}\right]_{+}+\left[-g_{1}-\varphi_{2}(L, t)-\varphi_{0}(L)\right]_{+}\right) \varphi_{t}(L, t) \\
\leq & \frac{\delta L}{2 \varepsilon}\left\|\varphi_{x t}\right\|^{2}+\frac{2 L}{\delta \varepsilon}\left\|\varphi_{x}\right\|^{2} .
\end{aligned}
$$

Therefore, using (2.21) and (2.1), (2.17) leads to

$$
\begin{aligned}
& \frac{1}{2} \frac{\mathrm{d}}{\mathrm{d} t}\left(\rho_{1}\left\|\varphi_{t}\right\|^{2}+\rho_{2}\left\|\psi_{t}\right\|^{2}+\rho_{1}\left\|\omega_{t}\right\|^{2}+k\left\|\varphi_{x}+\psi+l \omega\right\|^{2}+k_{0}\left\|\omega_{x}-l \varphi\right\|^{2}+b\left\|\psi_{x}\right\|^{2}\right) \\
& \quad+\zeta\left\|\varphi_{x t}+\psi_{t}+l \omega_{t}\right\|^{2}+\zeta\left\|\omega_{x t}-l \varphi_{t}\right\|^{2}+\zeta\left\|\psi_{x t}\right\|^{2} \\
& \quad \leq \frac{\delta L C}{2 \varepsilon}\left(\left\|\varphi_{x t}+\psi_{t}+l \omega_{t}\right\|^{2}+\left\|\omega_{x t}-l \varphi_{t}\right\|^{2}+\left\|\psi_{x t}\right\|^{2}\right)+\frac{2 L C}{\delta \varepsilon}\left(\left\|\varphi_{x}+\psi+l \omega\right\|^{2}+\left\|\omega_{x}-l \varphi\right\|^{2}+\left\|\psi_{x}\right\|^{2}\right) .
\end{aligned}
$$


Now, selecting $\delta=\frac{2 \varepsilon \zeta}{L C},(2.22)$ gives

$$
\begin{aligned}
& \frac{1}{2} \frac{\mathrm{d}}{\mathrm{d} t}\left(\rho_{1}\left\|\varphi_{t}\right\|^{2}+\rho_{2}\left\|\psi_{t}\right\|^{2}+\rho_{1}\left\|\omega_{t}\right\|^{2}+k\left\|\varphi_{x}+\psi+l \omega\right\|^{2}+k_{0}\left\|\omega_{x}-l \varphi\right\|^{2}+b\left\|\psi_{x}\right\|^{2}\right) \\
& \quad \leq C_{1}\left(\rho_{1}\left\|\varphi_{t}\right\|^{2}+\rho_{2}\left\|\psi_{t}\right\|^{2}+\rho_{1}\left\|\omega_{t}\right\|^{2}+k\left\|\varphi_{x}+\psi+l \omega\right\|^{2}+k_{0}\left\|\omega_{x}-l \varphi\right\|^{2}+b\left\|\psi_{x}\right\|^{2}\right) .
\end{aligned}
$$

Thus, by the Gronwall inequality, we get

$$
\rho_{1}\left\|\varphi_{t}\right\|^{2}+\rho_{2}\left\|\psi_{t}\right\|^{2}+\rho_{1}\left\|\omega_{t}\right\|^{2}+k\left\|\varphi_{x}+\psi+l \omega\right\|^{2}+k_{0}\left\|\omega_{x}-l \varphi\right\|^{2}+b\left\|\psi_{x}\right\|^{2}=0 .
$$

Thereby, due to (2.1), we have

$$
\left\|\varphi_{x}\right\|^{2}=\left\|\omega_{x}\right\|^{2}=\left\|\psi_{x}\right\|^{2}=0
$$

and so

$$
\varphi=\omega=\psi=0
$$

Hence, the uniqueness is established.

\section{Exponential Stability}

The exponential stability result is summarized in the following theorem.

Theorem 3.1. There exist two positive constants $C_{1}$ and $\eta$ such that the energy of the solution of (1.1)-(1.3) satisfies

$$
\mathcal{E}(t) \leq C_{1} \mathcal{E}(0) e^{-\eta t} \quad \forall t \geq 0
$$

Proof. Let

$$
\begin{aligned}
& I_{1}=\int_{0}^{L}\left(\rho_{1} \varphi_{t} \varphi+\rho_{2} \psi_{t} \psi+\rho_{1} \omega_{t} \omega\right) \mathrm{d} x, \\
& I_{2}=\frac{\zeta}{2}\left\|\varphi_{x}+\psi_{t}+l \omega_{t}\right\|^{2}+\frac{\zeta}{2}\left\|\omega_{x}-l \varphi\right\|^{2}+\frac{\zeta}{2}\left\|\psi_{x}\right\|^{2}
\end{aligned}
$$

and consider the functional $\mathscr{L}:=\beta \mathcal{E}(t)+I_{1}+I_{2}$ for a suitable choice of $\beta$.

First, using the Poincaré inequality and the estimate (2.1), we get

$$
\left|I_{1}\right| \leq K_{1} \mathcal{E}(t), \quad \forall t>0
$$

where $K_{1}$ is a positive constant that depends on $\rho_{1}, \rho_{2}, \rho_{1}, \zeta, C$. So, for $\beta$ sufficiently large, we have

$$
K_{2} \mathcal{E}(t) \leq \mathscr{L}(t) \leq K_{3} \mathcal{E}(t)
$$

where $K_{2}$ and $K_{3}$ are positive constants. Moreover, using the three equations of (1.1) we have

$$
\begin{aligned}
\frac{\mathrm{d}}{\mathrm{d} t} I_{1}= & \rho_{1}\left\|\varphi_{t}\right\|^{2}+\rho_{2}\left\|\psi_{t}\right\|^{2}+\rho_{1}\left\|\omega_{t}\right\|^{2}-k\left\|\varphi_{x}+\psi+l \omega\right\|^{2}-k_{0}\left\|w_{x}-l \varphi\right\|^{2}-b\left\|\psi_{x}\right\|^{2} \\
& -\frac{\zeta}{2} \frac{\mathrm{d}}{\mathrm{d} t}\left(\left\|\varphi_{x}+\psi+l \omega\right\|^{2}-\left\|w_{x}-l \varphi\right\|^{2}-\left\|\psi_{x}\right\|^{2}\right)-\frac{1}{\varepsilon}\left(\left[\varphi(L, t)-g_{2}\right]_{+}-\left[-g_{1}-\varphi(L, t)\right]_{+}\right) \varphi(L, t) .
\end{aligned}
$$


Now, let us estimate the last term in (3.2). In fact, we have

$$
\begin{aligned}
& -\frac{1}{\varepsilon}\left(\left[\varphi(L, t)-g_{2}\right]_{+}-\left[-g_{1}-\varphi(L, t)\right]_{+}\right) \varphi(L, t) \\
& \quad \leq-\frac{1}{\varepsilon}\left[\varphi(L, t)-g_{2}\right]_{+}\left(\varphi(L, t)-g_{2}\right)+\frac{1}{\varepsilon}\left[-g_{1}-\varphi(L, t)\right]_{+}\left(g_{1}+\varphi(L, t)\right) \\
& \quad \leq-\frac{1}{\varepsilon}\left(\left[\varphi(L, t)-g_{2}\right]_{+}^{2}+\left[-g_{1}-\varphi(L, t)\right]_{+}^{2}\right) .
\end{aligned}
$$

Therefore, using (3.3) in (3.2) we obtain

$$
\begin{aligned}
& \qquad \qquad \begin{aligned}
\frac{\mathrm{d}}{\mathrm{d} t} I_{1} \leq \rho_{1}\left\|\varphi_{t}\right\|^{2}+\rho_{2}\left\|\psi_{t}\right\|^{2}+\rho_{1}\left\|\omega_{t}\right\|^{2}-k\left\|\varphi_{x}+\psi+l \omega\right\|^{2}-k_{0}\left\|w_{x}-l \varphi\right\|^{2}-b\left\|\psi_{x}\right\|^{2} \\
\text { and so } \quad-\frac{\zeta}{2} \frac{\mathrm{d}}{\mathrm{d} t}\left(\left\|\varphi_{x}+\psi+l \omega\right\|^{2}-\left\|w_{x}-l \varphi\right\|^{2}-\left\|\psi_{x}\right\|^{2}\right)-\frac{1}{\varepsilon}\left(\left[\varphi(L, t)-g_{2}\right]_{+}^{2}+\left[-g_{1}-\varphi(L, t)\right]_{+}^{2}\right)
\end{aligned} \\
& \qquad \begin{array}{c}
\frac{\mathrm{d}}{\mathrm{d} t}\left(I_{1}+\frac{\zeta}{2}\left\|\varphi_{x}+\psi+l \omega\right\|^{2}+\frac{\zeta}{2}\left\|w_{x}-l \varphi\right\|^{2}+\frac{\zeta}{2}\left\|\psi_{x}\right\|^{2}\right) \leq \rho_{1}\left\|\varphi_{t}\right\|^{2}+\rho_{2}\left\|\psi_{t}\right\|^{2}+\rho_{1}\left\|\omega_{t}\right\|^{2}-k\left\|\varphi_{x}+\psi+l \omega\right\|^{2} \\
-k_{0}\left\|w_{x}-l \varphi\right\|^{2}-b\left\|\psi_{x}\right\|^{2}-\frac{1}{\varepsilon}\left(\left[\varphi(L, t)-g_{2}\right]_{+}^{2}\right. \\
+
\end{array}
\end{aligned}
$$

Next, thanks to (1.4) and (3.4), we get

$$
\begin{aligned}
\frac{\mathrm{d}}{\mathrm{d} t} \mathscr{L}(t) \leq & \beta\left(-\zeta\left\|\varphi_{x t}+\psi_{t}+l \omega_{t}\right\|^{2}-\zeta\left\|\psi_{x t}\right\|^{2}-\zeta \| \omega_{x t}-\left.l \varphi_{t}\right|^{2}\right) \\
& +\rho_{1}\left\|\varphi_{t}\right\|^{2}+\rho_{2}\left\|\psi_{t}\right\|^{2}+\rho_{1}\left\|\omega_{t}\right\|^{2}-k\left\|\varphi_{x}+\psi+l \omega\right\|^{2}-k_{0}\left\|w_{x}-l \varphi\right\|^{2} \\
& -b\left\|\psi_{x}\right\|^{2}-\frac{1}{\varepsilon}\left(\left[\varphi(L, t)-g_{2}\right]_{+}^{2}+\left[-g_{1}-\varphi(L, t)\right]_{+}^{2}\right) .
\end{aligned}
$$

Now, due to (2.1) and the Poincaré inequality, we have

$$
\begin{aligned}
\frac{\mathrm{d}}{\mathrm{d} t} \mathscr{L}(t) \leq & \beta\left(-\zeta c\left\|\varphi_{t}\right\|^{2}-\zeta c\left\|\psi_{t}\right\|^{2}-\zeta c\left\|\omega_{t}\right\|^{2}\right) \\
& +\rho_{1}\left\|\varphi_{t}\right\|^{2}+\rho_{2}\left\|\psi_{t}\right\|^{2}+\rho_{1}\left\|\omega_{t}\right\|^{2}-k\left\|\varphi_{x}+\psi+l \omega\right\|^{2}-k_{0}\left\|w_{x}-l \varphi\right\|^{2} \\
& -b\left\|\psi_{x}\right\|^{2}-\frac{1}{\varepsilon}\left(\left[\varphi(t, L)-g_{2}\right]_{+}^{2}+\left[-g_{1}-\varphi(t, L)\right]_{+}^{2}\right) .
\end{aligned}
$$

Thus,

$$
\begin{aligned}
\frac{\mathrm{d}}{\mathrm{d} t} \mathscr{L}(t) \leq & \left.\left(\rho_{1}-\beta \zeta c\right)\left\|\varphi_{t}\right\|^{2}+\left(\rho_{2}-\beta \zeta c\right)\left\|\psi_{t}\right\|^{2}+\left(\rho_{1}-\beta \zeta c\right)\left\|\omega_{t}\right\|^{2}\right) \\
& -k\left\|\varphi_{x}+\psi+l \omega\right\|^{2}-k_{0}\left\|w_{x}-l \varphi\right\|^{2}-b\left\|\psi_{x}\right\|^{2}-\frac{1}{\varepsilon}\left(\left[\varphi(L, t)-g_{2}\right]_{+}^{2}+\left[-g_{1}-\varphi(L, t)\right]_{+}^{2}\right) .
\end{aligned}
$$

Hence, for $\beta$ large enough, it follows that

$$
\frac{\mathrm{d}}{\mathrm{d} t} \mathscr{L}(t) \leq-c \mathcal{E}(t)
$$

Thus, due to (3.1), this leads to

$$
\frac{\mathrm{d}}{\mathrm{d} t} \mathscr{L}(t) \leq-\frac{c}{K_{3}} \mathscr{L}(t)
$$

and the proof is completed. 


\section{Numerical APPROXimation}

In this section, we will provide the numerical analysis of the problem described and studied, from a mathematical point of view, in the previous section.

For the spatial approximation of problem (2.2), we assume that the interval $[0, L]$ is divided into $M$ subintervals $a_{0}=0<a_{1}<\ldots<a_{M}=L$ of length $h=a_{i+1}-a_{i}=L / M$. Then, in order to approximate the variational spaces $H_{E}^{1}(I)$ and $H_{0}^{1}(I)$, we construct the finite dimensional spaces $S_{E}^{h} \subset H_{E}^{1}(I)$ and $S_{0}^{h} \subset H_{0}^{1}(I)$ given by

$$
\begin{aligned}
& S_{E}^{h}=\left\{\eta^{h} \in C([0, L]) ; \eta_{\mid\left[a_{i}, a_{i+1}\right]}^{h} \in P_{1}\left(\left[a_{i}, a_{i+1}\right]\right) \quad i=0, \ldots, M-1, \quad \eta^{h}(0)=0\right\}, \\
& S_{0}^{h}=\left\{\xi^{h} \in C([0, L]) ; \xi_{\left[a_{i}, a_{i+1}\right]}^{h_{1}} \in P_{1}\left(\left[a_{i}, a_{i+1}\right]\right) \quad i=0, \ldots, M-1, \quad \xi^{h}(0)=\xi^{h}(L)=0\right\},
\end{aligned}
$$

where $P_{1}\left(\left[a_{i}, a_{i+1}\right]\right)$ represents the space of polynomials of degree less or equal to 1 in the subinterval $\left[a_{i}, a_{i+1}\right]$; i.e. both finite element spaces are composed of continuous and piecewise affine functions. Here, $h>0$ denotes the spatial discretization parameter. Moreover, we assume that the discrete initial conditions, denoted by $\varphi_{h}^{0}$, $\tilde{\varphi}_{h}^{0}, \psi_{h}^{0}, \tilde{\psi}_{h}^{0}, \omega_{h}^{0}$ and $\tilde{\omega}_{h}^{0}$, are given by

$$
\varphi_{h}^{0}=P_{E}^{h} \varphi_{0}, \quad \tilde{\varphi}_{h}^{0}=P_{E}^{h} \varphi_{1}, \quad \psi_{h}^{0}=P_{E}^{h} \psi_{0}, \quad \tilde{\psi}_{h}^{0}=P_{E}^{h} \psi_{1}, \quad \omega_{h}^{0}=P_{0}^{h} \omega_{0}, \quad \tilde{\omega}_{h}^{0}=P_{0}^{h} \omega_{1} .
$$

Here, $P_{E}^{h}$ and $P_{0}^{h}$ are the classical finite element interpolation operators over $S_{E}^{h}$ and $S_{0}^{h}$, respectively (see [16]).

In order to provide the time discretization of problem (2.2), we consider a uniform partition of the time interval $[0, T]$, denoted by $0=t_{0}<t_{1}<\cdots<t_{N}=T$, with constant step size $\Delta t=T / N$ and nodes $t_{n}=n \Delta t$ for $n=0,1, \ldots, N$. For a continuous function $z(t)$, we use the notation $z^{n}=z\left(t_{n}\right)$ and $\tilde{z}=z_{t}$.

Therefore, using the backward Euler scheme in time, the fully discrete approximation of problem (2.2) is to find $\tilde{\varphi}_{h}^{n}, \tilde{\psi}_{h}^{n} \in S_{E}^{h}$ and $\tilde{\omega}_{h}^{n} \in S_{0}^{h}$ such that, for $n=1, \ldots, N$ and for all $\eta_{h}, \chi_{h} \in S_{E}^{h}$ and $\xi_{h} \in S_{0}^{h}$,

$$
\begin{aligned}
& \frac{\rho_{1}}{\Delta t}\left(\tilde{\varphi}_{h}^{n}-\tilde{\varphi}_{h}^{n-1}, \eta_{h}\right)+k\left(\varphi_{h x}^{n}+\psi_{h}^{n}+l \omega_{h}^{n}, \eta_{h x}\right)+\zeta\left(\tilde{\varphi}_{h x}^{n}+\tilde{\psi}_{h}^{n}+l \tilde{\omega}_{h}^{n}, \eta_{h x}\right)-k_{0} l\left(\omega_{h x}^{n}-l \varphi_{h}^{n}, \eta_{h}\right) \\
& \quad-\zeta l\left(\tilde{\omega}_{h x}^{n}-l \tilde{\varphi}_{h}^{n}, \eta_{h}\right)+\frac{1}{\varepsilon}\left(\left[\varphi_{h}^{n}(L, t)-g_{2}\right]_{+}-\left[-g_{1}-\varphi_{h}^{n}(L, t)\right]_{+}\right) \eta_{h}(L)=0, \\
& \frac{\rho_{2}}{\Delta t}\left(\tilde{\psi}_{h}^{n}-\tilde{\psi}_{h}^{n-1}, \chi_{h}\right)+b\left(\psi_{h x}^{n}, \chi_{h x}\right)+\zeta\left(\tilde{\psi}_{h x}^{n}, \chi_{h x}\right)+k\left(\varphi_{h x}^{n}+\psi_{h}^{n}+l \omega_{h}^{n}, \chi_{h}\right)+\zeta\left(\tilde{\varphi}_{h x}^{n}+\tilde{\psi}_{h}^{n}+l \tilde{\omega}_{h}^{n}, \chi_{h}\right)=0, \\
& \frac{\rho_{1}}{\Delta t}\left(\tilde{\omega}_{h}^{n}-\tilde{\omega}_{h}^{n-1}, \xi_{h}\right)+k_{0}\left(\omega_{h x}^{n}-l \varphi_{h}^{n}, \xi_{h x}\right)+\zeta\left(\tilde{\omega}_{h x}^{n}-l \tilde{\varphi}_{h}^{n}, \xi_{h x}\right)+k l\left(\varphi_{h x}^{n}+\psi_{h}^{n}+l \omega_{h}^{n}, \xi_{h}\right) \\
& \quad+\zeta l\left(\tilde{\varphi}_{h x}^{n}+\tilde{\psi}_{h}^{n}+l \tilde{\omega}_{h}^{n}, \xi_{h}\right)=0,
\end{aligned}
$$

where

$$
\tilde{\varphi}_{h}^{n}=\frac{\varphi_{h}^{n}-\varphi_{h}^{n-1}}{\Delta t}, \quad \tilde{\psi}_{h}^{n}=\frac{\psi_{h}^{n}-\psi_{h}^{n-1}}{\Delta t}, \quad \tilde{\omega}_{h}^{n}=\frac{\omega_{h}^{n}-\omega_{h}^{n-1}}{\Delta t}
$$

are approximations to $\tilde{\varphi}^{n}=\varphi_{t}\left(t_{n}\right), \tilde{\psi}^{n}=\psi_{t}\left(t_{n}\right)$ and $\tilde{\omega}^{n}=\omega_{t}\left(t_{n}\right)$, respectively.

The next result is a discrete version of the energy decay property (1.4) satisfied by the continuous solution.

Theorem 4.1. Let the discrete energy be given by

$$
\begin{aligned}
\mathcal{E}_{h}^{n}= & \frac{1}{2}\left(\rho_{1}\left(\left\|\tilde{\varphi}_{h}^{n}\right\|^{2}+\left\|\tilde{\omega}_{h}^{n}\right\|^{2}\right)+\rho_{2}\left\|\tilde{\psi}_{h}^{n}\right\|^{2}+b\left\|\psi_{h x}^{n}\right\|^{2}+k\left\|\varphi_{h x}^{n}+\psi_{h}^{n}+l \omega_{h}^{n}\right\|^{2}+k_{0}\left\|\omega_{h x}^{n}-l \varphi_{h}^{n}\right\|^{2}\right. \\
& \left.+\frac{1}{\varepsilon}\left(\left[\varphi_{h}^{n}(L, t)-g_{2}\right]_{+}^{2}+\left[-g_{1}-\varphi_{h}^{n}(L, t)\right]_{+}^{2}\right)\right) .
\end{aligned}
$$

Then, the decay property

$$
\frac{\mathcal{E}_{h}^{n}-\mathcal{E}_{h}^{n-1}}{\Delta t} \leq 0
$$

holds for $n=1,2, \ldots, N$. 
Proof. Taking $\eta_{h}=\tilde{\varphi}_{h}^{n}, \chi_{h}=\tilde{\psi}_{h}^{n}$ and $\xi_{h}=\tilde{\omega}_{h}^{n}$, it results that

$$
\begin{aligned}
& \frac{\rho_{1}}{2 \Delta t}\left(\left\|\tilde{\varphi}_{h}^{n}-\tilde{\varphi}_{h}^{n-1}\right\|^{2}+\left\|\tilde{\varphi}_{h}^{n}\right\|^{2}-\left\|\tilde{\varphi}_{h}^{n-1}\right\|^{2}\right)+k\left(\varphi_{h x}^{n}+\psi_{h}^{n}+l \omega_{h}^{n}, \tilde{\varphi}_{h x}^{n}\right) \\
& \quad+\zeta\left(\tilde{\varphi}_{h x}^{n}+\tilde{\psi}_{h}^{n}+l \tilde{\omega}_{h}^{n}, \tilde{\varphi}_{h x}^{n}\right)-k_{0} l\left(\omega_{h x}^{n}-l \varphi_{h}^{n}, \tilde{\varphi}_{h}^{n}\right)-\zeta l\left(\tilde{\omega}_{h x}^{n}-l \tilde{\varphi}_{h}^{n}, \tilde{\varphi}_{h}^{n}\right) \\
& \quad+\frac{1}{\varepsilon}\left(\left[\varphi_{h}^{n}(L, t)-g_{2}\right]_{+}-\left[-g_{1}-\varphi_{h}^{n}(L, t)\right]_{+}\right) \tilde{\varphi}_{h}^{n}(L)=0, \\
& \frac{\rho_{2}}{2 \Delta t}\left(\left\|\tilde{\psi}_{h}^{n}-\tilde{\psi}_{h}^{n-1}\right\|^{2}+\left\|\tilde{\psi}_{h}^{n}\right\|^{2}-\left\|\tilde{\psi}_{h}^{n-1}\right\|^{2}\right)+k\left(\varphi_{h x}^{n}+\psi_{h}^{n}+l \omega_{h}^{n}, \tilde{\psi}_{h}^{n}\right) \\
& \quad+\frac{b}{2 \Delta t}\left(\left\|\psi_{h x}^{n}-\psi_{h x}^{n-1}\right\|^{2}+\left\|\psi_{h x}^{n}\right\|^{2}-\left\|\psi_{h x}^{n-1}\right\|^{2}\right)+\zeta\left\|\tilde{\psi}_{h x}^{n}\right\|^{2}+\zeta\left(\tilde{\varphi}_{h x}^{n}+\tilde{\psi}_{h}^{n}+l \tilde{\omega}_{h}^{n}, \tilde{\psi}_{h}^{n}\right)=0,
\end{aligned}
$$

and

$$
\begin{aligned}
& \frac{\rho_{1}}{2 \Delta t}\left(\left\|\tilde{\omega}_{h}^{n}-\tilde{\omega}_{h}^{n-1}\right\|^{2}+\left\|\tilde{\omega}_{h}^{n}\right\|^{2}-\left\|\tilde{\omega}_{h}^{n-1}\right\|^{2}\right)+k l\left(\varphi_{h x}^{n}+\psi_{h}^{n}+l \omega_{h}^{n}, \tilde{\omega}_{h}^{n}\right) \\
& \quad+\zeta l\left(\tilde{\varphi}_{h x}^{n}+\tilde{\psi}_{h}^{n}+l \tilde{\omega}_{h}^{n}, \tilde{\omega}_{h}^{n}\right)+k_{0}\left(\omega_{h x}^{n}-l \varphi_{h}^{n}, \tilde{\omega}_{h x}^{n}\right)+\zeta\left(\tilde{\omega}_{h x}^{n}-l \tilde{\varphi}_{h}^{n}, \tilde{\omega}_{h x}^{n}\right)=0 .
\end{aligned}
$$

Thus, summing equations (4.6)-(4.8) and observing that

$$
k\left(\varphi_{h x}^{n}+\psi_{h}^{n}+l \omega_{h}^{n}, \tilde{\varphi}_{h x}^{n}+\tilde{\psi}_{h}^{n}+l \tilde{\omega}_{h}^{n}\right) \geq \frac{k}{2 \Delta t}\left(\left\|\varphi_{h x}^{n}+\psi_{h}^{n}+l \omega_{h}^{n}\right\|^{2}-\left\|\varphi_{h x}^{n-1}+\psi_{h}^{n-1}+l \omega_{h}^{n-1}\right\|^{2}\right)
$$

and that

$$
k_{0}\left(\omega_{h x}^{n}-l \varphi_{h}^{n}, \tilde{\omega}_{h x}^{n}-l \tilde{\varphi}_{h}^{n}\right) \geq \frac{k_{0}}{2 \Delta t}\left(\left\|\omega_{h x}^{n}-l \varphi_{h}^{n}\right\|^{2}-\left\|\omega_{h x}^{n-1}-l \varphi_{h}^{n-1}\right\|^{2}\right),
$$

we find

$$
\begin{aligned}
& \frac{\rho_{1}}{2 \Delta t}\left(\left\|\tilde{\varphi}_{h}^{n}\right\|^{2}-\left\|\tilde{\varphi}_{h}^{n-1}\right\|^{2}+\left\|\tilde{\omega}_{h}^{n}\right\|^{2}-\left\|\tilde{\omega}_{h}^{n-1}\right\|^{2}\right)+\frac{\rho_{2}}{2 \Delta t}\left(\left\|\tilde{\psi}_{h}^{n}\right\|^{2}-\left\|\tilde{\psi}_{h}^{n-1}\right\|^{2}\right)+\frac{b}{2 \Delta t}\left(\left\|\psi_{h x}^{n}\right\|^{2}-\left\|\psi_{h x}^{n-1}\right\|^{2}\right) \\
& \quad+\zeta\left\|\tilde{\psi}_{h x}^{n}\right\|^{2}+\frac{k}{2 \Delta t}\left(\left\|\varphi_{h x}^{n}+\psi_{h}^{n}+l \omega_{h}^{n}\right\|^{2}-\left\|\varphi_{h x}^{n-1}+\psi_{h}^{n-1}+l \omega_{h}^{n-1}\right\|^{2}\right) \\
& \quad+\frac{k_{0}}{2 \Delta t}\left(\left\|\omega_{h x}^{n}-l \varphi_{h}^{n}\right\|^{2}-\left\|\omega_{h x}^{n-1}-l \varphi_{h}^{n-1}\right\|^{2}\right)+\zeta\left\|\tilde{\varphi}_{h x}^{n}+\tilde{\psi}_{h}^{n}+l \tilde{\omega}_{h}^{n}\right\|^{2}+\zeta\left\|\tilde{\omega}_{h x}^{n}-l \tilde{\varphi}_{h}^{n}\right\|^{2} \\
& \quad+\frac{1}{\varepsilon}\left(\left[\varphi_{h}^{n}(L, t)-g_{2}\right]_{+}-\left[-g_{1}-\varphi_{h}^{n}(L, t)\right]_{+}\right) \tilde{\varphi}_{h}^{n}(L) \leq 0 .
\end{aligned}
$$

Now, we note that

$$
\begin{aligned}
& \frac{1}{\varepsilon}\left(\left[\varphi_{h}^{n}(L, t)-g_{2}\right]_{+}-\left[-g_{1}-\varphi_{h}^{n}(L, t)\right]_{+}\right) \tilde{\varphi}_{h}^{n}(L) \\
&=\frac{1}{\varepsilon}\left(\left[\varphi_{h}^{n}(L, t)-g_{2}\right]_{+}-\left[-g_{1}-\varphi_{h}^{n}(L, t)\right]_{+}\right)\left(\frac{\varphi_{h}^{n}(L)-\varphi_{h}^{n-1}(L)}{\Delta t}\right) \\
&=\frac{1}{\varepsilon \Delta t}\left(\left[\varphi_{h}^{n}(L, t)-g_{2}\right]_{+}\left(\varphi_{h}^{n}(L)+g_{2}-g_{2}-\varphi_{h}^{n-1}(L)\right)\right) \\
&\left.\quad-\frac{1}{\varepsilon \Delta t}\left[-g_{1}-\varphi_{h}^{n}(L, t)\right]_{+}\left(\varphi_{h}^{n}(L)+g_{1}-g_{1}-\varphi_{h}^{n-1}(L)\right)\right) \\
&=\frac{1}{\varepsilon \Delta t}\left(\left[\varphi_{h}^{n}(L, t)-g_{2}\right]_{+}^{2}-\left[\varphi_{h}^{n}(L)-g_{2}\right]_{+}\left(\varphi_{h}^{n-1}(L)-g_{2}\right)\right) \\
& \quad+\frac{1}{\varepsilon \Delta t}\left(\left[-g_{1}-\varphi_{h}^{n}(L, t)\right]_{+}^{2}-\left[-g_{1}-\varphi_{h}^{n}(L)\right]_{+}\left(-g_{1}-\varphi_{h}^{n-1}(L)\right)\right) \\
& \geq \frac{1}{\varepsilon \Delta t}\left(\left[\varphi_{h}^{n}(L, t)-g_{2}\right]_{+}^{2}-\left[\varphi_{h}^{n}(L)-g_{2}\right]_{+}\left[\varphi_{h}^{n-1}(L)-g_{2}\right]_{+}\right)
\end{aligned}
$$




$$
\begin{aligned}
& +\frac{1}{\varepsilon \Delta t}\left(\left[-g_{1}-\varphi_{h}^{n}(L, t)\right]_{+}^{2}-\left[-g_{1}-\varphi_{h}^{n}(L)\right]_{+}\left[-g_{1}-\varphi_{h}^{n-1}(L)\right]_{+}\right) \\
\geq & \frac{1}{2 \varepsilon \Delta t}\left(\left[\varphi_{h}^{n}(L)-g_{2}\right]_{+}^{2}-\left[\varphi_{h}^{n-1}(L)-g_{2}\right]_{+}^{2}\right) \\
& +\frac{1}{2 \varepsilon \Delta t}\left(\left[-g_{1}-\varphi_{h}^{n}(L)\right]_{+}^{2}-\left[-g_{1}-\varphi_{h}^{n-1}(L)\right]_{+}^{2}\right),
\end{aligned}
$$

which proves the result.

Now, we obtain some a priori error estimates on the numerical errors $\tilde{\varphi}^{n}-\tilde{\varphi}_{h}^{n}, \tilde{\psi}^{n}-\tilde{\psi}_{h}^{n}, \tilde{\omega}^{n}-\tilde{\omega}_{h}^{n}, \varphi^{n}-\varphi_{h}^{n}$, $\psi^{n}-\psi_{h}^{n}$ and $\omega^{n}-\omega_{h}^{n}$. We have the following.

Theorem 4.2. Let the assumptions of Theorem 2.2 hold. If we denote by $\left(\varphi^{n}, \tilde{\varphi}^{n}, \psi^{n}, \tilde{\psi}^{n}, \omega^{n}, \tilde{\omega}^{n}\right)$ the solution to problem (2.2) at time $t_{n}$ and by $\left(\varphi_{h}^{n}, \tilde{\varphi}_{h}^{n}, \psi_{h}^{n}, \tilde{\psi}_{h}^{n}, \omega_{h}^{n}, \tilde{\omega}_{h}^{n}\right)$ the solution to problem (4.4), then we have the following error estimates

$$
\begin{aligned}
& \max _{0 \leq n \leq N}\left\{\left\|\tilde{\varphi}^{n}-\tilde{\varphi}_{h}^{n}\right\|^{2}+\left\|\tilde{\psi}^{n}-\tilde{\psi}_{h}^{n}\right\|^{2}+\left\|\tilde{\omega}^{n}-\tilde{\omega}_{h}^{n}\right\|^{2}+\left\|\varphi^{n}-\varphi_{h}^{n}\right\|_{H^{1}(0, L)}^{2}+\left\|\psi^{n}-\psi_{h}^{n}\right\|_{H^{1}(0, L)}^{2}+\left\|\omega^{n}-\omega_{h}^{n}\right\|_{H^{1}(0, L)}^{2}\right\} \\
& \leq C \Delta t \sum_{j=1}^{N}\left(\left\|\tilde{\varphi}_{t}^{j}-\frac{1}{\Delta t}\left(\tilde{\varphi}^{j}-\tilde{\varphi}^{j-1}\right)\right\|^{2}+\left\|\tilde{\psi}_{t}^{j}-\frac{1}{\Delta t}\left(\tilde{\psi}^{j}-\tilde{\psi}^{j-1}\right)\right\|^{2}+\left\|\tilde{\omega}_{t}^{j}-\frac{1}{\Delta t}\left(\tilde{\omega}^{j}-\tilde{\omega}^{j-1}\right)\right\|^{2}\right. \\
&+\left\|\tilde{\varphi}^{j}-\eta_{h}^{j}\right\|_{H^{1}(0, L)}^{2}+\left\|\tilde{\psi}^{j}-\chi_{h}^{j}\right\|_{H^{1}(0, L)}^{2}+\left\|\tilde{\omega}^{j}-\xi_{h}^{j}\right\|_{H^{1}(0, L)}^{2}+\left\|\varphi_{t}^{j}-\frac{\varphi^{j}-\varphi^{j-1}}{\Delta t}\right\|_{H^{1}(0, L)}^{2} \\
&\left.+\left\|\psi_{t}^{j}-\frac{\psi^{j}-\psi^{j-1}}{\Delta t}\right\|_{H^{1}(0, L)}^{2}+\left\|\omega_{t}^{j}-\frac{\omega^{j}-\omega^{j-1}}{\Delta t}\right\|_{H^{1}(0, L)}^{2}\right)+C \max _{0 \leq n \leq N}\left\|\tilde{\varphi}^{n}-\eta_{h}^{n}\right\|^{2} \\
&+\frac{C}{\Delta t} \sum_{j=1}^{N-1}\left[\left\|\tilde{\varphi}^{j}-\eta_{h}^{j}-\left(\tilde{\varphi}^{j+1}-\eta_{h}^{j+1}\right)\right\|^{2}+\left\|\tilde{\psi}^{j}-\chi_{h}^{j}-\left(\tilde{\psi}^{j+1}-\chi_{h}^{j+1}\right)\right\|^{2}+\left\|\tilde{\omega}^{j}-\xi_{h}^{j}-\left(\tilde{\omega}^{j+1}-\xi_{h}^{j+1}\right)\right\|^{2}\right] \\
&+C \max _{0 \leq n \leq N}\left\|\tilde{\psi}^{n}-\chi_{h}^{n}\right\|^{2}+C \max _{0 \leq n \leq N}\left\|\tilde{\omega}^{n}-\xi_{h}^{n}\right\|^{2}+C\left(\left\|\varphi_{1}-\tilde{\varphi}_{h}^{0}\right\|^{2}+\left\|\psi_{1}-\tilde{\psi}_{h}^{0}\right\|^{2}+\left\|\omega_{1}-\tilde{\omega}_{h}^{0}\right\|^{2}\right. \\
&\left.+\left\|\varphi_{0}-\varphi_{h}^{0}\right\|_{H^{1}(0, L)}^{2}+\left\|\psi_{0}-\psi_{h}^{0}\right\|_{H^{1}(0, L)}^{2}+\left\|\omega_{0}-\omega_{h}^{0}\right\|_{H^{1}(0, L)}^{2}\right),
\end{aligned}
$$

for all $\eta_{h}=\left\{\eta_{h}^{j}\right\}_{j=0}^{N}, \chi_{h}=\left\{\chi_{h}^{j}\right\}_{j=0}^{N} \subset S_{E}^{h}$, and $\xi_{h}=\left\{\xi_{h}^{j}\right\}_{j=0}^{N} \subset S_{0}^{h}$.

Proof. Subtracting variational equations (2.2) at time $t_{n}$ for discrete test functions $\eta=\eta_{h}, \chi=\chi_{h}$ and $\xi=\xi_{h}$ and the corresponding discrete variational equations (4.4) we find, for all $\eta_{h}, \chi_{h} \in S_{E}^{h}, \xi_{h} \in S_{0}^{h}$,

$$
\begin{aligned}
& \rho_{1}\left(\tilde{\varphi}_{t}^{n}-\frac{1}{\Delta t}\left(\tilde{\varphi}_{h}^{n}-\tilde{\varphi}_{h}^{n-1}\right), \eta_{h}\right)+k\left(\varphi_{x}^{n}-\varphi_{h x}^{n}+\psi^{n}-\psi_{h}^{n}+l\left(\omega^{n}-\omega_{h}^{n}\right), \eta_{h x}\right)+\zeta\left(\tilde{\varphi}_{x}^{n}-\tilde{\varphi}_{h x}^{n}+\tilde{\psi}^{n}-\tilde{\psi}_{h}^{n}\right. \\
& \left.\quad+l\left(\tilde{\omega}^{n}-\tilde{\omega}_{h}^{n}\right), \eta_{h x}\right)-k_{0} l\left(\omega_{x}^{n}-\omega_{h x}^{n}-l\left(\varphi^{n}-\varphi_{h}^{n}\right), \eta_{h}\right)-\zeta l\left(\tilde{\omega}_{x}-\tilde{\omega}_{h x}^{n}-l\left(\tilde{\varphi}^{n}-\tilde{\varphi}_{h}^{n}\right), \eta_{h}\right) \\
& \quad+\frac{1}{\varepsilon}\left(\left[\varphi^{n}(L, t)-g_{2}\right]_{+}-\left[-g_{1}-\varphi^{n}(L, t)\right]_{+}-\left[\varphi_{h}^{n}(L, t)-g_{2}\right]_{+}+\left[-g_{1}-\varphi_{h}^{n}(L, t)\right]_{+}\right) \eta_{h}(L)=0, \\
& \rho_{2}\left(\tilde{\psi}_{t}^{n}-\frac{1}{\Delta t}\left(\tilde{\psi}_{h}^{n}-\tilde{\psi}_{h}^{n-1}\right), \chi_{h}\right)+b\left(\psi_{x}^{n}-\psi_{h x}^{n}, \chi_{h x}\right)+\zeta\left(\tilde{\psi}_{x}^{n}-\tilde{\psi}_{h x}^{n}, \chi_{h x}\right) \\
& \quad+k\left(\varphi_{x}^{n}-\varphi_{h x}^{n}+\psi^{n}-\psi_{h}^{n}+l\left(\omega^{n}-\omega_{h}^{n}\right), \chi_{h}\right)+\zeta\left(\tilde{\varphi}_{x}^{n}-\tilde{\varphi}_{h x}^{n}+\tilde{\psi}^{n}-\tilde{\psi}_{h}^{n}+l\left(\tilde{\omega}-\tilde{\omega}_{h}^{n}\right), \chi_{h}\right)=0, \\
& \rho_{1}\left(\tilde{\omega}_{t}^{n}-\frac{1}{\Delta t}\left(\tilde{\omega}_{h}^{n}-\tilde{\omega}_{h}^{n-1}\right), \xi_{h}\right)+k_{0}\left(\omega_{x}^{n}-\omega_{h x}^{n}-l\left(\varphi^{n}-\varphi_{h}^{n}\right), \xi_{h x}\right)+\zeta\left(\tilde{\omega}_{x}^{n}-\tilde{\omega}_{h x}^{n}-l\left(\tilde{\varphi}^{n}-\tilde{\varphi}_{h}^{n}\right), \xi_{h x}\right) \\
& \quad+k l\left(\varphi_{x}^{n}-\varphi_{h x}^{n}+\psi^{n}-\psi_{h}^{n}+l\left(\omega^{n}-\omega_{h}^{n}\right), \xi_{h}\right)+\zeta l\left(\tilde{\varphi}_{x}^{n}-\tilde{\varphi}_{h x}^{n}+\tilde{\psi}^{n}-\tilde{\psi}_{h}^{n}+l\left(\tilde{\omega}^{n}-\tilde{\omega}_{h}^{n}\right), \xi_{h}\right)=0,
\end{aligned}
$$


and therefore,

$$
\begin{aligned}
& \rho_{1}\left(\tilde{\varphi}_{t}^{n}-\frac{1}{\Delta t}\left(\tilde{\varphi}_{h}^{n}-\tilde{\varphi}_{h}^{n-1}\right), \tilde{\varphi}^{n}-\tilde{\varphi}_{h}^{n}\right)+k\left(\varphi_{x}^{n}-\varphi_{h x}^{n}+\psi^{n}-\psi_{h}^{n}+l\left(\omega^{n}-\omega_{h}^{n}\right), \tilde{\varphi}_{x}^{n}-\tilde{\varphi}_{h x}^{n}\right) \\
& +\zeta\left(\tilde{\varphi}_{x}^{n}-\tilde{\varphi}_{h x}^{n}+\tilde{\psi}^{n}-\tilde{\psi}_{h}^{n}+l\left(\tilde{\omega}^{n}-\tilde{\omega}_{h}^{n}\right), \tilde{\varphi}_{x}^{n}-\tilde{\varphi}_{h x}^{n}\right) \\
& -k_{0} l\left(\omega_{x}^{n}-\omega_{h x}^{n}-l\left(\varphi^{n}-\varphi_{h}^{n}\right), \tilde{\varphi}^{n}-\tilde{\varphi}_{h}^{n}\right)-\zeta l\left(\tilde{\omega}_{x}-\tilde{\omega}_{h x}^{n}-l\left(\tilde{\varphi}^{n}-\tilde{\varphi}_{h}^{n}\right), \tilde{\varphi}^{n}-\tilde{\varphi}_{h}^{n}\right) \\
& +\frac{1}{\varepsilon}\left(\left[\varphi^{n}(L, t)-g_{2}\right]_{+}-\left[-g_{1}-\varphi^{n}(L, t)\right]_{+}-\left[\varphi_{h}^{n}(L, t)-g_{2}\right]_{+}+\left[-g_{1}-\varphi_{h}^{n}(L, t)\right]_{+}\right)\left(\tilde{\varphi}^{n}-\tilde{\varphi}_{h}^{n}\right)(L) \\
& =\rho_{1}\left(\tilde{\varphi}_{t}\left(t_{n}\right)-\frac{1}{\Delta t}\left(\tilde{\varphi}_{h}^{n}-\tilde{\varphi}_{h}^{n-1}\right), \tilde{\varphi}^{n}-\eta_{h}\right)+k\left(\varphi_{x}^{n}-\varphi_{h x}^{n}+\psi^{n}-\psi_{h}^{n}+l\left(\omega^{n}-\omega_{h}^{n}\right),\left(\tilde{\varphi}^{n}-\eta_{h}\right)_{x}\right) \\
& +\zeta\left(\tilde{\varphi}_{x}^{n}-\tilde{\varphi}_{h x}^{n}+\tilde{\psi}^{n}-\tilde{\psi}_{h}^{n}+l\left(\tilde{\omega}^{n}-\tilde{\omega}_{h}^{n}\right),\left(\tilde{\varphi}^{n}-\eta_{h}\right)_{x}\right) \\
& -k_{0} l\left(\omega_{x}^{n}-\omega_{h x}^{n}-l\left(\varphi^{n}-\varphi_{h}^{n}\right), \tilde{\varphi}^{n}-\eta_{h}\right)-\zeta l\left(\tilde{\omega}_{x}-\tilde{\omega}_{h x}^{n}-l\left(\tilde{\varphi}^{n}-\tilde{\varphi}_{h}^{n}\right), \tilde{\varphi}^{n}-\eta_{h}\right) \\
& +\frac{1}{\varepsilon}\left(\left[\varphi^{n}(L, t)-g_{2}\right]_{+}-\left[-g_{1}-\varphi^{n}(L, t)\right]_{+}-\left[\varphi_{h}^{n}(L, t)-g_{2}\right]_{+}+\left[-g_{1}-\varphi_{h}^{n}(L, t)\right]_{+}\right)\left(\tilde{\varphi}^{n}-\eta_{h}\right)(L), \\
& \rho_{2}\left(\tilde{\psi}_{t}^{n}-\frac{1}{\Delta t}\left(\tilde{\psi}_{h}^{n}-\tilde{\psi}_{h}^{n-1}\right), \tilde{\psi}^{n}-\tilde{\psi}_{h}^{n}\right)+b\left(\psi_{x}^{n}-\psi_{h x}^{n}, \tilde{\psi}_{x}^{n}-\tilde{\psi}_{h x}^{n}\right)+\zeta\left(\tilde{\psi}_{x}^{n}-\tilde{\psi}_{h x}^{n}, \tilde{\psi}_{x}^{n}-\tilde{\psi}_{h x}^{n}\right) \\
& +k\left(\varphi_{x}^{n}-\varphi_{h x}^{n}+\psi^{n}-\psi_{h}^{n}+l\left(\omega^{n}-\omega_{h}^{n}\right), \tilde{\psi}^{n}-\tilde{\psi}_{h}^{n}\right)+\zeta\left(\tilde{\varphi}_{x}^{n}-\tilde{\varphi}_{h x}^{n}+\tilde{\psi}^{n}-\tilde{\psi}_{h}^{n}+l\left(\tilde{\omega}-\tilde{\omega}_{h}^{n}\right), \tilde{\psi}^{n}-\tilde{\psi}_{h}^{n}\right) \\
& =\rho_{2}\left(\tilde{\psi}_{t}^{n}-\frac{1}{\Delta t}\left(\tilde{\psi}_{h}^{n}-\tilde{\psi}_{h}^{n-1}\right), \tilde{\psi}^{n}-\chi_{h}\right)+b\left(\psi_{x}^{n}-\psi_{h x}^{n},\left(\tilde{\psi}^{n}-\chi_{h}\right)_{x}\right)+\zeta\left(\tilde{\psi}_{x}^{n}-\tilde{\psi}_{h x}^{n},\left(\tilde{\psi}^{n}-\chi_{h}\right)_{x}\right) \\
& +k\left(\varphi_{x}^{n}-\varphi_{h x}^{n}+\psi^{n}-\psi_{h}^{n}+l\left(\omega^{n}-\omega_{h}^{n}\right), \tilde{\psi}^{n}-\chi_{h}\right)+\zeta\left(\tilde{\varphi}_{x}^{n}-\tilde{\varphi}_{h x}^{n}+\tilde{\psi}^{n}-\tilde{\psi}_{h}^{n}+l\left(\tilde{\omega}-\tilde{\omega}_{h}^{n}\right), \tilde{\psi}^{n}-\chi_{h}\right), \\
& \rho_{1}\left(\tilde{\omega}_{t}^{n}-\frac{1}{\Delta t}\left(\tilde{\omega}_{h}^{n}-\tilde{\omega}_{h}^{n-1}\right), \tilde{\omega}^{n}-\tilde{\omega}_{h}^{n}\right)+k_{0}\left(\omega_{x}^{n}-\omega_{h x}^{n}-l\left(\varphi^{n}-\varphi_{h}^{n}\right), \tilde{\omega}_{x}^{n}-\tilde{\omega}_{h x}^{n}\right) \\
& +\zeta\left(\tilde{\omega}_{x}^{n}-\tilde{\omega}_{h x}^{n}-l\left(\tilde{\varphi}^{n}-\tilde{\varphi}_{h}^{n}\right), \tilde{\omega}_{x}^{n}-\tilde{\omega}_{h x}^{n}\right)+k l\left(\varphi_{x}^{n}-\varphi_{h x}^{n}+\psi^{n}-\psi_{h}^{n}+l\left(\omega^{n}-\omega_{h}^{n}\right), \tilde{\omega}^{n}-\tilde{\omega}_{h}^{n}\right) \\
& +\zeta l\left(\tilde{\varphi}_{x}^{n}-\tilde{\varphi}_{h x}^{n}+\tilde{\psi}^{n}-\tilde{\psi}_{h}^{n}+l\left(\tilde{\omega}^{n}-\tilde{\omega}_{h}^{n}\right), \tilde{\omega}^{n}-\tilde{\omega}_{h}^{n}\right) \\
& =\rho_{1}\left(\tilde{\omega}_{t}^{n}-\frac{1}{\Delta t}\left(\tilde{\omega}_{h}^{n}-\tilde{\omega}_{h}^{n-1}\right), \tilde{\omega}^{n}-\xi_{h}\right)+k_{0}\left(\omega_{x}^{n}-\omega_{h x}^{n}-l\left(\varphi^{n}-\varphi_{h}^{n}\right),\left(\omega^{n}-\xi_{h}\right)_{x}\right) \\
& +\zeta\left(\tilde{\omega}_{x}^{n}-\tilde{\omega}_{h x}^{n}-l\left(\tilde{\varphi}^{n}-\tilde{\varphi}_{h}^{n}\right),\left(\tilde{\omega}^{n}-\xi_{h}\right)_{x}\right)+k l\left(\varphi_{x}^{n}-\varphi_{h x}^{n}+\psi^{n}-\psi_{h}^{n}+l\left(\omega^{n}-\omega_{h}^{n}\right), \tilde{\omega}^{n}-\xi_{h}\right) \\
& +\zeta l\left(\tilde{\varphi}_{x}^{n}-\tilde{\varphi}_{h x}^{n}+\tilde{\psi}^{n}-\tilde{\psi}_{h}^{n}+l\left(\tilde{\omega}^{n}-\tilde{\omega}_{h}^{n}\right), \tilde{\omega}^{n}-\xi_{h}\right) .
\end{aligned}
$$

Now, we observe that

$$
\begin{aligned}
& \left(\tilde{\varphi}_{t}^{n}-\frac{1}{\Delta t}\left(\tilde{\varphi}_{h}^{n}-\tilde{\varphi}_{h}^{n-1}\right), \tilde{\varphi}^{n}-\tilde{\varphi}_{h}^{n}\right) \geq\left(\tilde{\varphi}_{t}^{n}-\frac{1}{\Delta t}\left(\tilde{\varphi}^{n}-\tilde{\varphi}^{n-1}\right), \tilde{\varphi}^{n}-\tilde{\varphi}_{h}^{n}\right)+\frac{1}{\Delta t}\left(\left\|\tilde{\varphi}^{n}-\tilde{\varphi}_{h}^{n}\right\|-\left\|\tilde{\varphi}^{n-1}-\tilde{\varphi}_{h}^{n-1}\right\|\right) \\
& \left(\tilde{\psi}_{t}^{n}-\frac{1}{\Delta t}\left(\tilde{\psi}_{h}^{n}-\tilde{\psi}_{h}^{n-1}\right), \tilde{\psi}^{n}-\tilde{\psi}_{h}^{n}\right) \geq\left(\tilde{\psi}_{t}^{n}-\frac{1}{\Delta t}\left(\tilde{\psi}^{n}-\tilde{\psi}^{n-1}\right), \tilde{\psi}^{n}-\tilde{\psi}_{h}^{n}\right)+\frac{1}{\Delta t}\left(\left\|\tilde{\psi}^{n}-\tilde{\psi}_{h}^{n}\right\|-\left\|\tilde{\psi}^{n-1}-\tilde{\psi}_{h}^{n-1}\right\|\right) \\
& \left(\tilde{\omega}_{t}^{n}-\frac{1}{\Delta t}\left(\tilde{\omega}_{h}^{n}-\tilde{\omega}_{h}^{n-1}\right), \tilde{\omega}^{n}-\tilde{\omega}_{h}^{n}\right) \geq\left(\tilde{\omega}_{t}^{n}-\frac{1}{\Delta t}\left(\tilde{\omega}^{n}-\tilde{\omega}^{n-1}\right), \tilde{\omega}^{n}-\tilde{\omega}_{h}^{n}\right)+\frac{1}{\Delta t}\left(\left\|\tilde{\omega}^{n}-\tilde{\omega}_{h}^{n}\right\|-\left\|\tilde{\omega}^{n-1}-\tilde{\omega}_{h}^{n-1}\right\|\right) \\
& \left(\varphi_{x}^{n}-\varphi_{h x}^{n}, \tilde{\varphi}_{x}^{n}-\tilde{\varphi}_{h x}^{n}\right) \geq\left(\varphi_{x}^{n}-\varphi_{h x}^{n}, \varphi_{x t}^{n}-\frac{\varphi_{x}^{n}-\varphi_{x}^{n-1}}{\Delta t}\right)+\frac{1}{\Delta t}\left(\left\|\varphi_{x}^{n}-\varphi_{h x}^{n}\right\|^{2}-\left\|\varphi_{x}^{n-1}-\varphi_{h x}^{n-1}\right\|^{2}\right) \\
& \left(\psi_{x}^{n}-\psi_{h x}^{n}, \tilde{\psi}_{x}^{n}-\tilde{\psi}_{h x}^{n}\right) \geq\left(\psi_{x}^{n}-\psi_{h x}^{n}, \psi_{x t}^{n}-\frac{\psi_{x}^{n}-\psi_{x}^{n-1}}{\Delta t}\right)+\frac{1}{\Delta t}\left(\left\|\psi_{x}^{n}-\psi_{h x}^{n}\right\|^{2}-\left\|\psi_{x}^{n-1}-\psi_{h x}^{n-1}\right\|^{2}\right)
\end{aligned}
$$




$$
\begin{aligned}
& \left(\omega_{x}^{n}-\omega_{h x}^{n}, \tilde{\omega}_{x}^{n}-\tilde{\omega}_{h x}^{n}\right) \geq\left(\omega_{x}^{n}-\omega_{h x}^{n}, \omega_{x t}^{n}-\frac{\omega_{x}^{n}-\omega_{x}^{n-1}}{\Delta t}\right)+\frac{1}{\Delta t}\left(\left\|\omega_{x}^{n}-\omega_{h x}^{n}\right\|^{2}-\left\|\omega_{x}^{n-1}-\omega_{h x}^{n-1}\right\|^{2}\right), \\
& \left(\varphi^{n}-\varphi_{h}^{n}, \tilde{\varphi}^{n}-\tilde{\varphi}_{h}^{n}\right) \geq\left(\varphi^{n}-\varphi_{h}^{n}, \varphi_{t}^{n}-\frac{\varphi^{n}-\varphi^{n-1}}{\Delta t}\right)+\frac{1}{\Delta t}\left(\left\|\varphi^{n}-\varphi_{h}^{n}\right\|^{2}-\left\|\varphi^{n-1}-\varphi_{h}^{n-1}\right\|^{2}\right), \\
& \left(\psi^{n}-\psi_{h}^{n}, \tilde{\psi}^{n}-\tilde{\psi}_{h}^{n}\right) \geq\left(\psi^{n}-\psi_{h}^{n}, \psi_{t}^{n}-\frac{\psi^{n}-\psi^{n-1}}{\Delta t}\right)+\frac{1}{\Delta t}\left(\left\|\psi^{n}-\psi_{h}^{n}\right\|^{2}-\left\|\psi^{n-1}-\psi_{h}^{n-1}\right\|^{2}\right), \\
& \left(\omega^{n}-\omega_{h}^{n}, \tilde{\omega}^{n}-\tilde{\omega}_{h}^{n}\right) \geq\left(\omega^{n}-\omega_{h}^{n}, \omega_{t}^{n}-\frac{\omega^{n}-\omega^{n-1}}{\Delta t}\right)+\frac{1}{\Delta t}\left(\left\|\omega^{n}-\omega_{h}^{n}\right\|^{2}-\left\|\omega^{n-1}-\omega_{h}^{n-1}\right\|^{2}\right), \\
& \left|\frac{1}{\varepsilon}\left(\left[\varphi^{n}(L, t)-g_{2}\right]_{+}-\left[-g_{1}-\varphi^{n}(L, t)\right]_{+}-\left[\varphi_{h}^{n}(L, t)-g_{2}\right]_{+}+\left[-g_{1}-\varphi_{h}^{n}(L, t)\right]_{+}\right) \eta(L)\right| \\
& \quad \leq C\left(\left\|\varphi^{n}-\varphi_{h}^{n}\right\|_{H^{1}(0, L)}^{2}+\|\eta\|_{H^{1}(0, L)}^{2}\right)
\end{aligned}
$$

and summing up the previous three equations, using Young's inequality (2.15) several times and Cauchy-Schwarz inequality we find that, for all $\eta_{h}, \chi_{h} \in S_{E}^{h}, \xi_{h} \in S_{0}^{h}$,

$$
\begin{aligned}
\frac{1}{\Delta t} & \left(\left\|\tilde{\varphi}^{n}-\tilde{\varphi}_{h}^{n}\right\|-\left\|\tilde{\varphi}^{n-1}-\tilde{\varphi}_{h}^{n-1}\right\|\right)+\frac{1}{\Delta t}\left(\left\|\tilde{\psi}^{n}-\tilde{\psi}_{h}^{n}\right\|-\left\|\tilde{\psi}^{n-1}-\tilde{\psi}_{h}^{n-1}\right\|\right) \\
& +\frac{1}{\Delta t}\left(\left\|\tilde{\omega}^{n}-\tilde{\omega}_{h}^{n}\right\|-\left\|\tilde{\omega}^{n-1}-\tilde{\omega}_{h}^{n-1}\right\|\right)+\frac{1}{\Delta t}\left(\left\|\varphi_{x}^{n}-\varphi_{h x}^{n}\right\|^{2}-\left\|\varphi_{x}^{n-1}-\varphi_{h x}^{n-1}\right\|^{2}\right) \\
& +\frac{1}{\Delta t}\left(\left\|\psi_{x}^{n}-\psi_{h x}^{n}\right\|^{2}-\left\|\psi_{x}^{n-1}-\psi_{h x}^{n-1}\right\|^{2}\right)+\frac{1}{\Delta t}\left(\left\|\omega_{x}^{n}-\omega_{h x}^{n}\right\|^{2}-\left\|\omega_{x}^{n-1}-\omega_{h x}^{n-1}\right\|^{2}\right) \\
& +\frac{1}{\Delta t}\left(\left\|\varphi^{n}-\varphi_{h}^{n}\right\|^{2}-\left\|\varphi^{n-1}-\varphi_{h}^{n-1}\right\|^{2}\right)+\frac{1}{\Delta t}\left(\left\|\psi^{n}-\psi_{h}^{n}\right\|^{2}-\left\|\psi^{n-1}-\psi_{h}^{n-1}\right\|^{2}\right) \\
& +\frac{1}{\Delta t}\left(\left\|\omega^{n}-\omega_{h}^{n}\right\|^{2}-\left\|\omega^{n-1}-\omega_{h}^{n-1}\right\|^{2}\right) \\
\leq & C\left(\left\|\tilde{\varphi}_{t}^{n}-\frac{1}{\Delta t}\left(\tilde{\varphi}^{n}-\tilde{\varphi}^{n-1}\right)\right\|^{2}+\left\|\tilde{\psi}_{t}^{n}-\frac{1}{\Delta t}\left(\tilde{\psi}^{n}-\tilde{\psi}^{n-1}\right)\right\|^{2}\right. \\
& +\left\|\tilde{\omega}_{t}^{n}-\frac{1}{\Delta t}\left(\tilde{\omega}^{n}-\tilde{\omega}^{n-1}\right)\right\|^{2}+\left\|\tilde{\varphi}^{n}-\eta_{h}\right\|_{H^{1}(0, L)}^{2} \\
& +\left\|\tilde{\psi}^{n}-\chi_{h}\right\|_{H^{1}(0, L)}^{2}+\left\|\tilde{\omega}^{n}-\xi_{h}\right\|_{H^{1}(0, L)}^{2}+\left\|\varphi_{x t}^{n}-\frac{\varphi_{x}^{n}-\varphi_{x}^{n-1}}{\Delta t}\right\|^{2} \\
& +\left\|\psi_{x t}^{n}-\frac{\psi_{x}^{n}-\psi_{x}^{n-1}}{\Delta t}\right\|^{2}+\left\|\omega_{x t}^{n}-\frac{\omega_{x}^{n}-\omega_{x}^{n-1}}{\Delta t}\right\|^{2} \\
& +\left(\frac{1}{\Delta t}\left(\tilde{\varphi}^{n}-\tilde{\varphi}^{n-1}-\left(\tilde{\varphi}_{h}^{n}-\tilde{\varphi}_{h}^{n-1}\right)\right), \tilde{\varphi}^{n}-\eta_{h}\right)+\left\|\varphi_{t}^{n}-\frac{\varphi^{n}-\varphi^{n-1}}{\Delta t}\right\|^{2} \\
& +\left(\frac{1}{\Delta t}\left(\tilde{\psi}^{n}-\tilde{\psi}^{n-1}-\left(\tilde{\psi}_{h}^{n}-\tilde{\psi}_{h}^{n-1}\right)\right), \tilde{\psi}^{n}-\chi_{h}\right)+\left\|\psi_{t}^{n}-\frac{\psi^{n}-\psi^{n-1}}{\Delta t}\right\|^{2} \\
& \left.+\left(\frac{1}{\Delta t}\left(\tilde{\omega}^{n}-\tilde{\omega}^{n-1}-\left(\tilde{\omega}_{h}^{n}-\tilde{\omega}_{h}^{n-1}\right)\right), \tilde{\omega}^{n}-\xi_{h}\right)+\left\|\omega_{t}^{n}-\frac{\omega^{n}-\omega^{n-1}}{\Delta t}\right\|^{2}\right)
\end{aligned}
$$

Multiplying the above estimates by $\Delta t$ and summing up to $n$, it follows that, for all $\eta_{h}=\left\{\eta_{h}^{j}\right\}_{j=0}^{n}, \chi_{h}=$ $\left\{\chi_{h}^{j}\right\}_{j=0}^{n} \subset S_{E}^{h}, \xi_{h}=\left\{\xi_{h}^{j}\right\}_{j=0}^{n} \subset S_{0}^{h}$,

$$
\begin{aligned}
& \left\|\tilde{\varphi}^{n}-\tilde{\varphi}_{h}^{n}\right\|+\left\|\tilde{\psi}^{n}-\tilde{\psi}_{h}^{n}\right\|+\left\|\tilde{\omega}^{n}-\tilde{\omega}_{h}^{n}\right\|+\left\|\varphi^{n}-\varphi_{h}^{n}\right\|_{H^{1}(0, L)}^{2}+\left\|\psi^{n}-\psi_{h}^{n}\right\|_{H^{1}(0, L)}^{2}+\left\|\omega^{n}-\omega_{h}^{n}\right\|_{H^{1}(0, L)}^{2} \\
& \quad \leq C \Delta t \sum_{j=1}^{n}\left(\left\|\tilde{\varphi}_{t}^{j}-\frac{1}{\Delta t}\left(\tilde{\varphi}^{j}-\tilde{\varphi}^{j-1}\right)\right\|^{2}+\left\|\tilde{\psi}_{t}^{j}-\frac{1}{\Delta t}\left(\tilde{\psi}^{j}-\tilde{\psi}^{j-1}\right)\right\|^{2}+\left\|\tilde{\omega}_{t}^{j}-\frac{1}{\Delta t}\left(\tilde{\omega}^{j}-\tilde{\omega}^{j-1}\right)\right\|^{2}\right.
\end{aligned}
$$




$$
\begin{aligned}
& +\left\|\tilde{\varphi}^{j}-\eta_{h}^{j}\right\|_{H^{1}(0, L)}^{2}+\left\|\tilde{\psi}^{j}-\chi_{h}^{j}\right\|_{H^{1}(0, L)}^{2}+\left\|\tilde{\omega}^{j}-\xi_{h}^{j}\right\|_{H^{1}(0, L)}^{2}+\left\|\varphi_{t}^{j}-\frac{\varphi^{j}-\varphi^{j-1}}{\Delta t}\right\|_{H^{1}(0, L)}^{2} \\
& +\left\|\psi_{t}^{j}-\frac{\psi^{j}-\psi^{j-1}}{\Delta t}\right\|_{H^{1}(0, L)}^{2}+\left\|\omega_{t}^{j}-\frac{\omega^{j}-\omega^{j-1}}{\Delta t}\right\|_{H^{1}(0, L)}^{2}+\left(\frac{1}{\Delta t}\left(\tilde{\varphi}^{j}-\tilde{\varphi}^{j-1}-\left(\tilde{\varphi}_{h}^{j}-\tilde{\varphi}_{h}^{j-1}\right)\right), \tilde{\varphi}^{j}-\eta_{h}^{j}\right) \\
& \left.+\left(\frac{1}{\Delta t}\left(\tilde{\psi}^{j}-\tilde{\psi}^{j-1}-\left(\tilde{\psi}_{h}^{j}-\tilde{\psi}_{h}^{j-1}\right)\right), \tilde{\psi}^{j}-\chi_{h}^{j}\right)+\left(\frac{1}{\Delta t}\left(\tilde{\omega}^{j}-\tilde{\omega}^{j-1}-\left(\tilde{\omega}_{h}^{j}-\tilde{\omega}_{h}^{j-1}\right)\right), \tilde{\omega}^{j}-\xi_{h}^{j}\right)\right) \\
& +C\left(\left\|\varphi^{0}-\tilde{\varphi}_{h}^{0}\right\|+\left\|\psi^{1}-\tilde{\psi}_{h}^{0}\right\|+\left\|\omega^{1}-\tilde{\omega}_{h}^{0}\right\|+\left\|\varphi_{x}^{0}-\varphi_{h x}^{0}\right\|^{2}+\left\|\psi_{x}^{0}-\psi_{h x}^{0}\right\|^{2}+\left\|\omega_{x}^{0}-\omega_{h x}^{0}\right\|^{2}\right) .
\end{aligned}
$$

Taking into account that

$$
\begin{aligned}
& \Delta t \sum_{j=1}^{n}\left(\frac{1}{\Delta t}\left(\tilde{\varphi}^{j}-\tilde{\varphi}^{j-1}-\left(\tilde{\varphi}_{h}^{j}-\tilde{\varphi}_{h}^{j-1}\right)\right), \tilde{\varphi}^{j}-\eta_{h}^{j}\right)=\sum_{j=1}^{n}\left(\tilde{\varphi}^{j}-\tilde{\varphi}_{h}^{j}-\left(\tilde{\varphi}^{j-1}-\tilde{\varphi}_{h}^{j-1}\right), \tilde{\varphi}^{j}-\eta_{h}^{j}\right) \\
& \quad=\left(\tilde{\varphi}^{n}-\tilde{\varphi}_{h}^{n}, \tilde{\varphi}^{n}-\eta_{h}^{n}\right)+\left(\tilde{\varphi}_{h}^{0}-\varphi_{1}, \tilde{\varphi}^{1}-\eta_{h}^{1}\right)+\sum_{j=1}^{n-1}\left(\tilde{\varphi}^{j}-\tilde{\varphi}_{h}^{j}, \tilde{\varphi}^{j}-\eta_{h}^{j}-\left(\tilde{\varphi}^{j+1}-\eta_{h}^{j+1}\right)\right)
\end{aligned}
$$

where we omit the similar estimates in $\tilde{\psi}$ and $\tilde{\omega}$ for the sake of simplicity in the writing, using a discrete version of Gronwall's inequality, the result follows.

The error estimates provided in the above theorem can be used to obtain the convergence order of the approximations introduced in problem (4.4). For instance, under suitable regularity conditions, the linear convergence is deduced and summarized in the following.

Corollary 4.3. If we assume the following additional regularity conditions:

$$
\varphi, \psi, \omega \in C^{1}\left([0, T] ; H^{2}(I)\right) \cap H^{3}\left(0, T ; L^{2}(I)\right) \cap H^{2}\left(0, T ; H^{1}(I)\right),
$$

then there exists a positive constant $C>0$, independent of discretization parameters $h$ and $\Delta t$, such that

$$
\begin{gathered}
\max _{0 \leq n \leq N}\left\{\left\|\tilde{\varphi}^{n}-\tilde{\varphi}_{h}^{n}\right\|+\left\|\tilde{\psi}^{n}-\tilde{\psi}_{h}^{n}\right\|+\left\|\tilde{\omega}^{n}-\tilde{\omega}_{h}^{n}\right\|+\left\|\varphi^{n}-\varphi_{h}^{n}\right\|_{H^{1}(0, L)}\right. \\
\left.+\left\|\psi^{n}-\psi_{h}^{n}\right\|_{H^{1}(0, L)}+\left\|\omega^{n}-\omega_{h}^{n}\right\|_{H^{1}(0, L)}\right\} \leq C(h+\Delta t) .
\end{gathered}
$$

The proof of the above corollary is shown using classical results on the approximation by finite elements (see [16]) and the estimates like (see [28]),

$$
\frac{C}{\Delta t} \sum_{j=1}^{N-1}\left\|\tilde{\varphi}^{j}-\eta_{h}^{j}-\left(\tilde{\varphi}^{j+1}-\eta_{h}^{j+1}\right)\right\|^{2} \leq C h^{2}\|\varphi\|_{H^{2}\left(0, T ; H^{1}(0, L)\right)}^{2} .
$$

\section{NUMERICAL EXPERIMENTS}

In this section we present the procedure used to find the numerical solution and the results of some numerical simulations.

To solve the nonlinear problem (4.4) we use the iterative process:

$$
\begin{aligned}
& \frac{\rho_{2}}{\Delta t}\left(\tilde{\psi}_{h}^{n, j}-\tilde{\psi}_{h}^{n-1}, \eta_{h}\right)+b\left(\psi_{h x}^{n, j}, \eta_{h x}\right)+\zeta\left(\tilde{\psi}_{h x}^{n, j}, \eta_{h x}\right)+k\left(\varphi_{h x}^{n, j-1}+\psi_{h}^{n, j}+l \omega_{h}^{n, j-1}, \eta_{h}\right) \\
& \quad+\zeta\left(\tilde{\varphi}_{h x}^{n, j-1}+\tilde{\psi}_{h}^{n, j}+l \tilde{\omega}_{h}^{n, j-1}, \eta_{h}\right)=0, \\
& \frac{\rho_{1}}{\Delta t}\left(\tilde{\omega}_{h}^{n, j}-\tilde{\omega}_{h}^{n-1}, \xi_{h}\right)+k_{0}\left(\omega_{h x}^{n, j}-l \varphi_{h}^{n, j-1}, \xi_{h x}\right)+\gamma_{0}\left(\tilde{\omega}_{h x}^{n, j}-l \tilde{\varphi}_{h}^{n, j-1}, \xi_{h x}\right)+k l\left(\varphi_{h x}^{n, j-1}+\psi_{h}^{n, j}+l \omega_{h}^{n}, \xi_{h}\right)
\end{aligned}
$$




$$
\begin{aligned}
& \quad+\gamma_{1} l\left(\tilde{\varphi}_{h x}^{n, j-1}+\tilde{\psi}_{h}^{n, j}+l \tilde{\omega}_{h}^{n, j}, \xi_{h}\right)=0, \\
& \frac{\rho_{1}}{\Delta t}\left(\tilde{\varphi}_{h}^{n, j}-\tilde{\varphi}_{h}^{n-1}, \zeta_{h}\right)+k\left(\varphi_{h x}^{n, j}+\psi_{h}^{n, j}+l \omega_{h}^{n, j}, \zeta_{h x}\right)+\gamma_{1}\left(\tilde{\varphi}_{h x}^{n, j}+\tilde{\psi}_{h}^{n, j}+l \tilde{\omega}_{h}^{n, j}, \zeta_{h x}\right) \\
& \quad-k_{0} l\left(\omega_{h x}^{n, j}-l \varphi_{h}^{n, j}, \zeta_{h}\right)-\gamma_{0} l\left(\tilde{\omega}_{h x}^{n, j}-l \tilde{\varphi}_{h}^{n, j}, \zeta_{h}\right)+g\left(\varphi_{h}^{n, j-1}, \varphi^{n, j}\right)(L)=0,
\end{aligned}
$$

where

$$
g\left(\varphi_{h}^{n, j-1}, \varphi_{h}^{n, j}\right)(L)= \begin{cases}\frac{1}{\varepsilon}\left(\varphi_{h}^{n, l}(L)-g_{2}\right) & \text { if } \varphi_{h}^{n, j-1}(L) \geq g_{2}, \\ 0 & \text { if }-g_{1}<\varphi_{h}^{n, j-1}(1)<g_{2}, \\ \frac{1}{\varepsilon}\left(g_{1}+\varphi_{h}^{n, j}(L)\right) & \text { if } \varphi_{h}^{n, j-1}(L) \leq-g_{1},\end{cases}
$$

and, for $j=1,2, \ldots$,

$$
\psi_{h}^{n, j}=\psi_{h}^{n-1}+\Delta t \tilde{\psi}_{h}^{n, j}, \quad \omega_{h}^{n, j}=\omega_{h}^{n-1}+\Delta t \tilde{\omega}_{h}^{n, j}, \quad \varphi_{h}^{n, j}=\varphi_{h}^{n-1}+\Delta t \tilde{\varphi}_{h}^{n, j} .
$$

Hence, three uncoupled linear systems of algebraic equations, which have a unique solution, are solved. First, we compute $\tilde{\psi}_{h}^{n, j}$, then $\tilde{\omega}_{h}^{n, j}$ and finally $\tilde{\varphi}_{h}^{n, j}$.

The iterations are started with $\tilde{\psi}_{h}^{n, 0}=\tilde{\psi}_{h}^{n-1}, \tilde{\omega}_{h}^{n, 0}=\tilde{\omega}_{h}^{n-1}, \tilde{\varphi}_{n}^{n, 0}=\tilde{\varphi}_{h}^{n-1}$ and a tolerance of $10^{-7}$ is used to stop the process. In all the simulations, we choose a circular beam with radius of curvature $R=1$ with $g_{1}=0.01, g_{2}=0.02, \varepsilon=0.001, \rho_{1}=1, \rho_{2}=2, k=1, k_{0}=2, b=1$ and $\zeta=0.1$.

\subsection{Experiment 1: long time evolution}

In this experiment, the length of the beam is $L=0.5 \pi$ and the discretization parameters are $h=0.5 \pi / 100$ and $\Delta t=10^{-4}$. The initial conditions are

$$
\begin{aligned}
& \varphi_{0}(x)=g_{1}\left((2 x / \pi)^{2}-4 x / \pi\right), \quad \varphi_{1}(x)=20 x(x-0.5 \pi)^{2}, \\
& \psi_{0}=\psi_{1}=\omega_{0}=0, \quad \omega_{1}=x^{3}-0.5 \pi x^{2},
\end{aligned}
$$

and we note that, at initial time, the beam is in contact with the lower obstacle.

The long time evolution of $\psi$ and $\varphi$ at contact point $x=L$ is presented in Figure 2. An oscillatory behavior is observed with the beam getting in contact with both stops during some time interval. As the system evolves, contact is lost. The spatial position of the beam, obtained taking into account the longitudinal and transverse displacements, is shown in Figure 3 where we see that, at time $t=80$, the configuration is close to the reference configuration, that is, a quarter circle. The results at point $x=0.5 \mathrm{~L}$ are displayed in Figure 4 .

In Figure 5 the discrete energy is seen and exponential decay rate seems to be achieved after time $t=10$.

\subsection{Experiment 2: numerical convergence}

Next, we examine numerically the error estimate for a beam with length $L=1$ considering the academic problem:

$$
\begin{aligned}
& \rho_{1} \varphi_{t t}-k\left(\varphi_{x}+l \omega+\psi\right)_{x}-\zeta\left(\varphi_{x}+l w+\psi\right)_{x t}-k_{0} l\left(\omega_{x}-l \varphi\right)-\zeta l\left(w_{x}-l \varphi\right)_{t}=f_{1}, \\
& \rho_{2} \psi_{t t}-b \psi_{x x}-\zeta \psi_{x x t}+k\left(\varphi_{x}+\psi+l \omega\right)+\zeta\left(\varphi_{x}+l \omega+\psi\right)_{t}=f_{2}, \\
& \rho_{1} \omega_{t t}-k_{0}\left(\omega_{x}-l \varphi\right)_{x}-\zeta\left(\omega_{x}-l \varphi\right)_{x t}+k l\left(\varphi_{x}+\psi+l \omega\right)+\zeta l\left(\varphi_{x}+\psi+l \omega\right)_{t}=f_{3}, \\
& \sigma=k\left(\varphi_{x}+l \omega+\psi\right)+\zeta\left(\varphi_{x}+l w+\psi\right)_{t}+f_{4}
\end{aligned}
$$

with exact solution:

$$
\begin{aligned}
& \varphi(x, t)=-g_{2}\left(x^{2}-2 x\right) t^{2} / \sqrt{2} \\
& \psi(x, t)=0.5 t^{2}\left(0.5 x^{2}-x\right)
\end{aligned}
$$



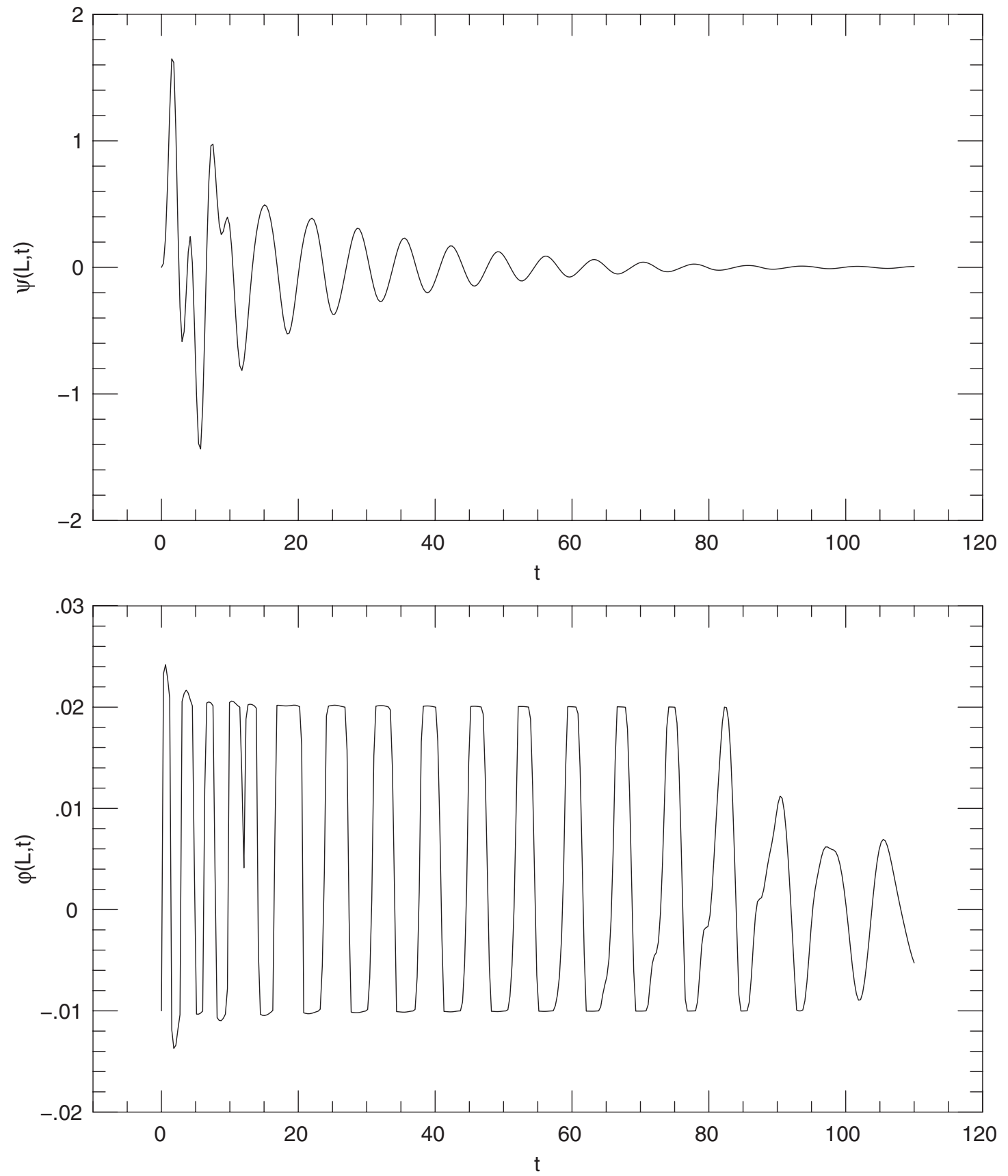

Figure 2. The evolution in time of $\varphi$ and $\psi$ at the contact point $L=0.5 \pi$. 

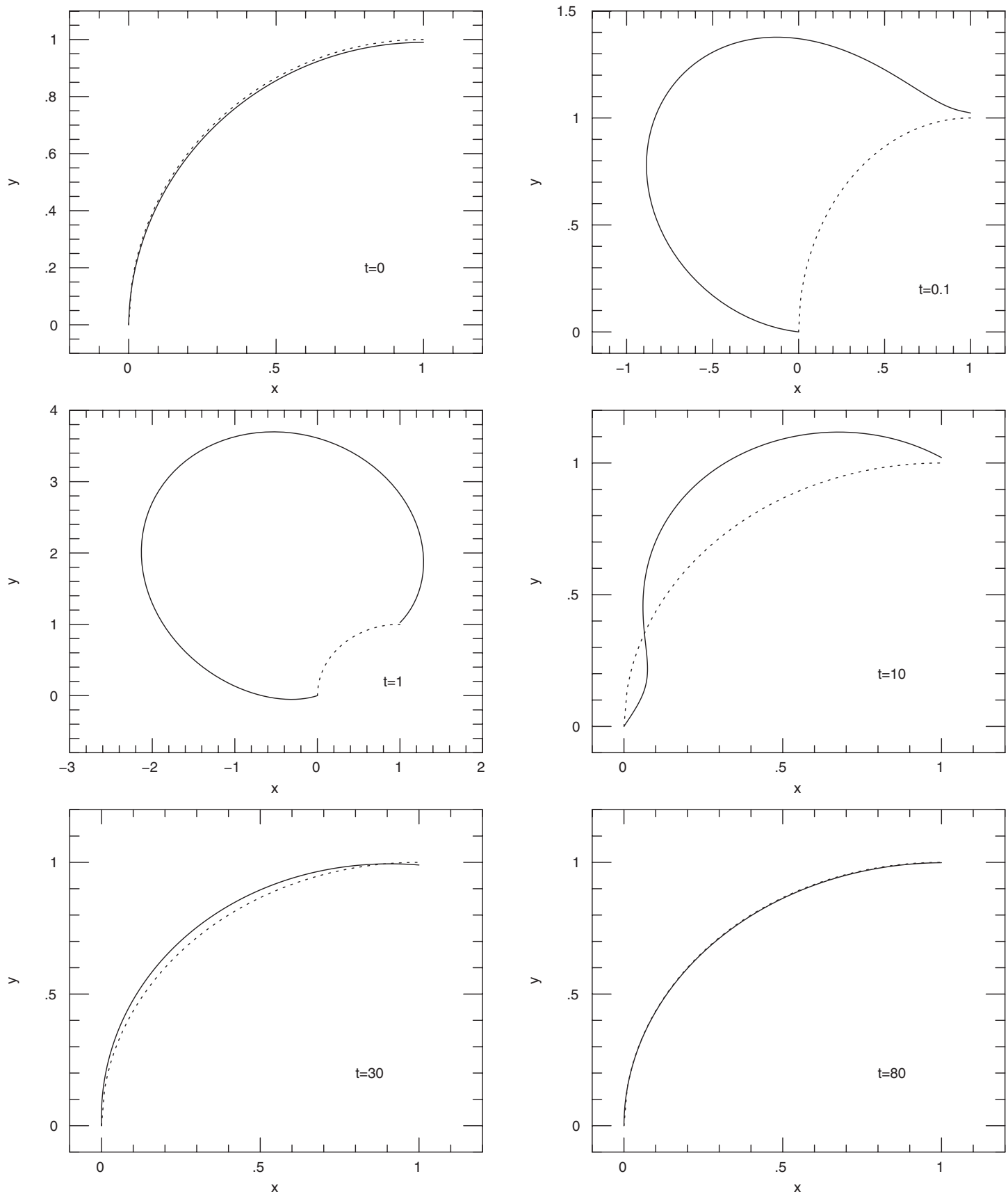

FiguRE 3. The beam's configuration when time increases. The traced line represents the reference configuration. 

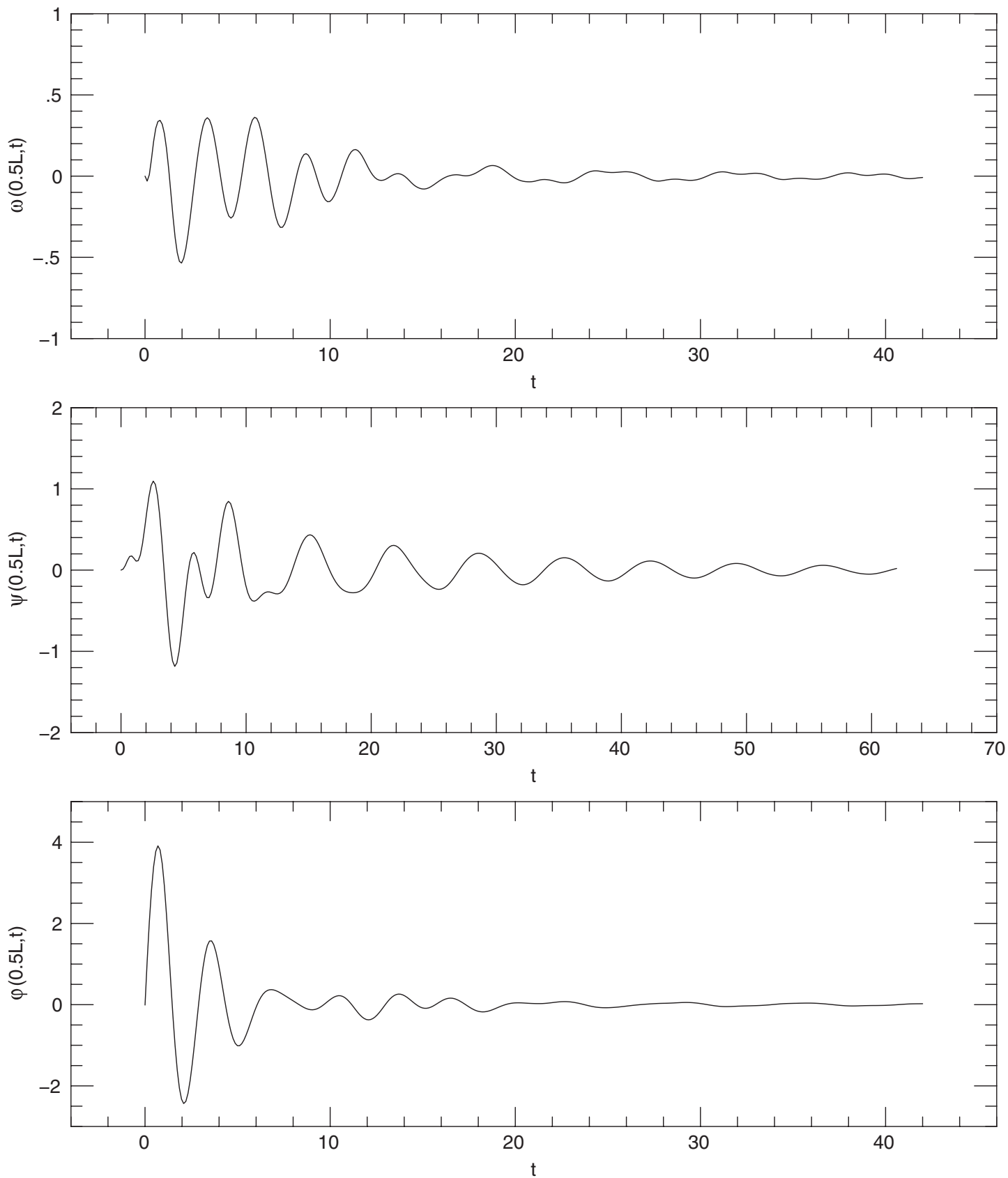

Figure 4. The evolution in time of $\varphi, \psi$ and $\omega$ at $x=0.5 L=0.25 \pi$. 

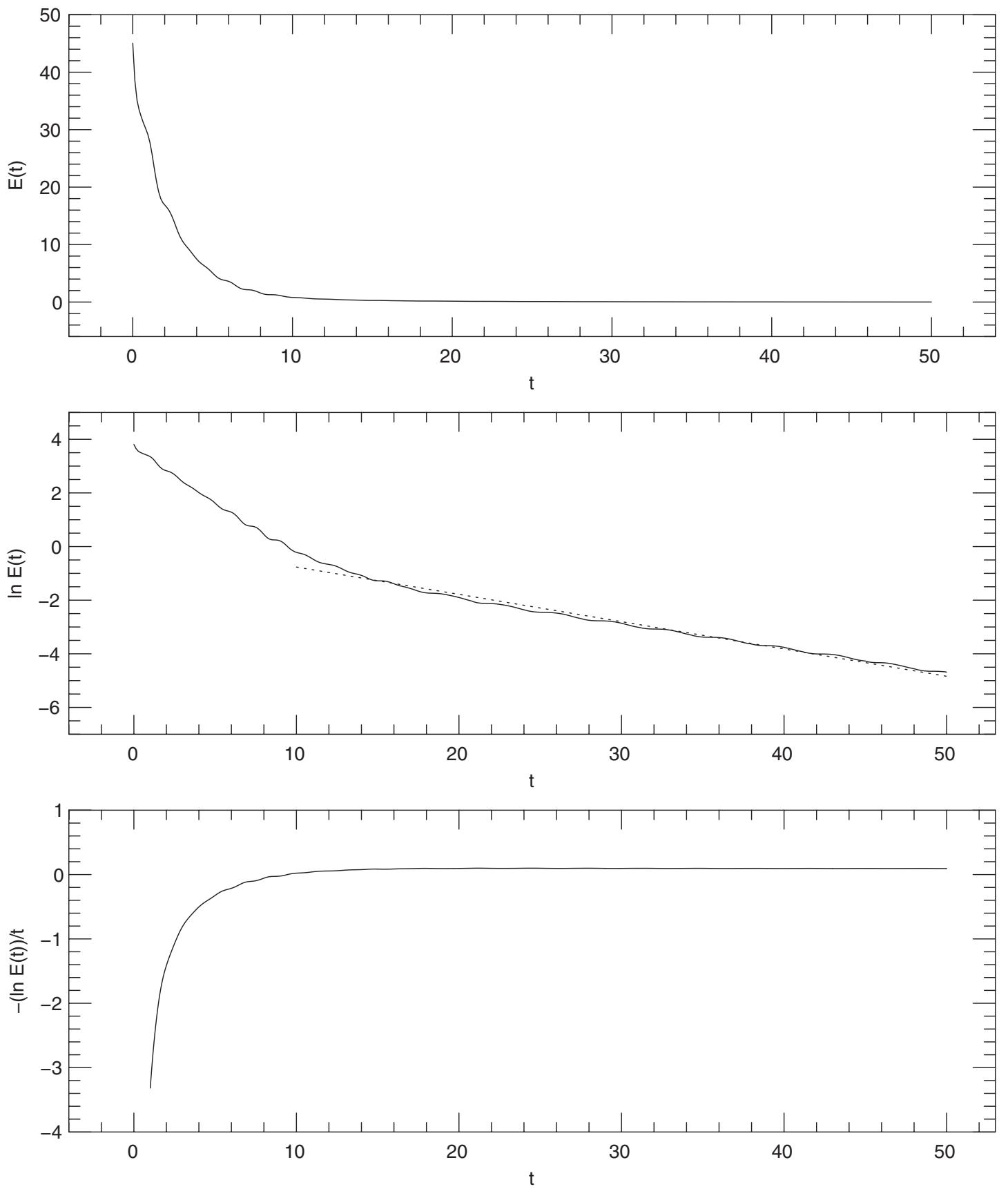

Figure 5. The time evolution of the energy. The traced line is a linear regression for $t>10$. 
TABLE 1. Computed errors when $t_{n}=1.5$.

\begin{tabular}{lll}
\hline \hline$M$ & $\Delta t$ & Error \\
\hline 40 & $2.50 \times 10^{-3}$ & $4.922 \times 10^{-2}$ \\
80 & $1.25 \times 10^{-3}$ & $2.498 \times 10^{-2}$ \\
160 & $6.25 \times 10^{-4}$ & $1.262 \times 10^{-2}$ \\
320 & $3.125 \times 10^{-4}$ & $6.349 \times 10^{-3}$ \\
640 & $1.5625 \times 10^{-4}$ & $3.185 \times 10^{-3}$ \\
1280 & $7.8125 \times 10^{-5}$ & $1.595 \times 10^{-3}$ \\
\hline
\end{tabular}

$$
\begin{aligned}
\omega(x, t) & =t x^{2}(x-1)^{2}, \\
\sigma(x, t) & =-\frac{1}{\varepsilon}\left[g_{2}\left(t^{2} / \sqrt{2}\right)-1\right]_{+},
\end{aligned}
$$

and functions $f_{1}, f_{2}, f_{3}, f_{4}$ calculated from the given solution. Note that, when $t^{2} \geq \sqrt{2}$, the beam is in contact with the upper obstacle.

The computed errors given by

$$
\left\|\tilde{\varphi}^{n}-\tilde{\varphi}_{h}^{n}\right\|+\left\|\tilde{\psi}^{n}-\tilde{\psi}_{h}^{n}\right\|+\left\|\tilde{\omega}^{n}-\tilde{\omega}_{h}^{n}\right\|+\left\|\varphi^{n}-\varphi_{h}^{n}\right\|_{H^{1}(0, L)}+\left\|\psi^{n}-\psi_{h}^{n}\right\|_{H^{1}(0, L)}+\left\|\omega^{n}-\omega_{h}^{n}\right\|_{H^{1}(0, L)} .
$$

at $t_{n}=1.5$ are displayed in Table 1 . We observe that the errors decrease by a factor of approximately 2 when the discretization parameters are halved.

Acknowledgements. The work of M.I.M. Copetti was partially supported by the Brazilian institution CNPq (grant 304709/2017-4). The work of J.R. Fernández was partially supported by the research project PGC2018-096696-B-I00 (Ministerio de Ciencia, Innovación y Universidades, Spain) with the participation of FEDER.

\section{REFERENCES}

[1] F. Alabau Boussouira, J.E. Muñoz Rivera and D. da S. Almeida Júnior, Stability to weak dissipative Bresse system. J. Math. Anal. Appl. 374 (2011) 481-498.

[2] M.O. Alves, L.H. Fatori, M.A. Jorge Silva and R.N. Monteiro, Stability and optimality of decay rate for a weakly dissipative Bresse system. Math. Methods Appl. Sci. 38 (2015) 898-908.

[3] K.T. Andrews, M. Shillor and S. Wright, On the dynamic vibrations of an elastic beam in frictional contact with a rigid obstacle. J. Elasticity 42 (1996) 1-30.

[4] H. Antes and P.D. Panagiotopoulos, The boundary integral approach to static and dynamic contact problems. In: Vol. 108 of International Series of Numerical Mathematics. Equality and inequality methods. Birkhäuser Verlag, Basel (1992).

[5] M. Aouadi and M.I.M. Copetti, Analytical and numerical results for a dynamic contact problem with two stops in thermoelastic diffusion theory. ZAMM Z. Angew. Math. Mech. 96 (2016) 361-384.

[6] M. Aouadi, M.I.M. Copetti and J.R. Fernández, A contact problem in thermoviscoelastic diffusion theory with second sound. ESAIM: M2AN $\mathbf{5 1}$ (2017) 759-796.

[7] D.N. Arnold, A.L. Madureira and S. Zhang, On the range of applicability of the Reissner-Mindlin and Kirchhoff-Love plate bending models. J. Elasticity 67 (2002) 171-185.

[8] C. Bernardi and M. I. M. Copetti, Discretization of a nonlinear dynamic thermoviscoelastic Timoshenko beam model. $Z A M M$ Z. Angew. Math. Mech. 97 (2017) 532-549.

[9] A. Berti and M.G. Naso, Unilateral dynamic contact of two viscoelastic beams. Q. Appl. Math. 69 (2011) $477-507$.

[10] A. Berti, M.I.M. Copetti, J.R. Fernández and M.G. Naso, A dynamic thermoviscoelastic contact problem with the second sound effect. J. Math. Anal. Appl. 421 (2015) 1163-1195.

[11] A. Berti, J.E. Muñoz Rivera and M.G. Naso, A contact problem for a thermoelastic Timoshenko beam. Z. Angew. Math. Phys. 66 (2015) 1969-1986.

[12] G. Bonfanti, J.E. Muñoz Rivera and M.G. Naso, Global existence and exponential stability for a contact problem between two thermoelastic beams. J. Math. Anal. Appl. 345 (2008) 186-202.

[13] G. Bonfanti, M. Fabrizio, J.E. Muñoz Rivera and M.G. Naso, On the energy decay for a thermoelastic contact problem involving heat transfer. J. Thermal Stresses 33 (2010) 1049-1065. 
[14] J.E.C. Bresse, Cours de mécanique appliquée, professé a l'École des ponts et chaussées, par M. Bresse. Gauthier-Villars, Paris (1865-1868).

[15] M. Campo, M.I.M. Copetti and J.R. Fernández, Dynamic vibrations of a damageable viscoelastic beam in contact with two stops. Numer. Methods Part. Differ. Equ. 29 (2013) 647-666.

[16] P.G. Ciarlet, Basic error estimates for elliptic problems. In: Vol. II of Handbook of numerical Analysis. Handb. Numer. Anal. II. North-Holland, Amsterdam (1991) 17-351. MR 1115237.

[17] M.I.M. Copetti, Finite element approximation to a quasi-static thermoelastic problem to the contact of two rods. Appl. Numer. Math. 44 (2003) 31-47.

[18] M.I.M. Copetti, Numerical approximation of dynamic deformations of a thermoviscoelastic rod against an elastic obstacle. M2AN Math. Model. Numer. Anal. 38 (2004) 691-706.

[19] M.I.M. Copetti and D.A. French, Numerical approximation and error control for a thermoelastic contact problem. Appl. Numer. Math. 55 (2005) 439-457.

[20] A.D. de Pater and J.J. Kalker, The Mechanics of the Contact Between Deformable Bodies. Delft University Press, Delft (1975).

[21] G. Duvaut and J.-L. Lions, Inequalities in Mechanics and Physics. Springer-Verlag, Berlin (1976).

[22] C. Eck, J. Jarušek and M. Krbec, Unilateral contact problems. In: Vol. 270 of Pure and Applied Mathematics (Boca Raton). Variational Methods and Existence Theorems. Chapman \& Hall/CRC, Boca Raton, FL (2005).

[23] T. El Arwadi and W. Youssef, On the stabilization of the Bresse beam with Kelvin-Voigt damping. To appear in: Appl. Math. Opt. https://doi.org/10.1007/s00245-019-09611-z (2019).

[24] T. EL Arwadi, M.I.M. Copetti and W. Youssef, On the theoretical and numerical stability of the thermoviscoelastic Bresse system. ZAMM Z. Angew. Math. Mech. 99 (2019) e201800207.

[25] L.H. Fatori and J.E. Muñoz Rivera, Rates of decay to weak thermoelastic Bresse system. IMA J. Appl. Math. 75 (2010) 881-904.

[26] M. Frémond, Contact with adhesion. Topics in Nonsmooth Mechanics. Birkhäuser, Basel (1988) 157-185.

[27] M. Frémond, Non-Smooth Thermomechanics. Springer-Verlag, Berlin (2002).

[28] W. Han, M. Shillor and M. Sofonea, Variational and numerical analysis of a quasistatic viscoelastic problem with normal compliance, friction and damage. J. Comput. Appl. Math. 137 (2001) 377-398.

[29] N. Kikuchi and J.T. Oden, Contact problems in elasticity: a study of variational inequalities and finite element methods. In: Vol. 8 of SIAM Studies in Applied Mathematics. Society for Industrial and Applied Mathematics (SIAM), Philadelphia, PA (1988).

[30] J.U. Kim, A one-dimensional dynamic contact problem in linear viscoelasticity. Math. Methods Appl. Sci. 13 (1990) 55-79.

[31] K.L. Kuttler and M. Shillor, Vibrations of a beam between two stops. Dyn. Contin. Discrete Impuls. Syst. Ser. B Appl. Algorithms 8 (2001) 93-110.

[32] A. Labuschagne, N.F.J. van Rensburg and A.J. van der Merwe, Comparison of linear beam theories. Math. Comput. Modelling 49 (2009) 20-30.

[33] Z. Liu and B. Rao, Energy decay rate of the thermoelastic Bresse system. Z. Angew. Math. Phys. 60 (2009) 54-69.

[34] J.E. Muñoz Rivera and S. Jiang, The thermoelastic and viscoelastic contact of two rods. J. Math. Anal. Appl. 217 (1998) 423-458.

[35] M. Nakao and J.E. Muñoz Rivera, The contact poblem in thermoviscoelastic materials. J. Math. Anal. Appl. 264 (2001) $522-545$.

[36] F.G. Pfeiffer, Applications of unilateral multibody dynamics. Phil. Trans. R. Soc. Lond. A 359 (2001) $2609-2628$.

[37] M.L. Santos and D. da S. Almeida Júnior, Numerical exponential decay to dissipative Bresse system. J. Appl. Math. (2010) 848620.

[38] M.E. Stavroulaki and G.E. Stavroulakis, Unilateral contact applications using fem software. Int. J. Appl. Math. Comput. Sci. 12 (2002) 115-125.

[39] A. Wehbe and W. Youssef, Exponential and polynomial stability of an elastic Bresse system with two locally distributed feedbacks. J. Math. Phys. 51 (2010) 103523.

[40] W. Youssef, Contrôle et stabilisation de systèmes élastiques couplés, Thesis (2009). 